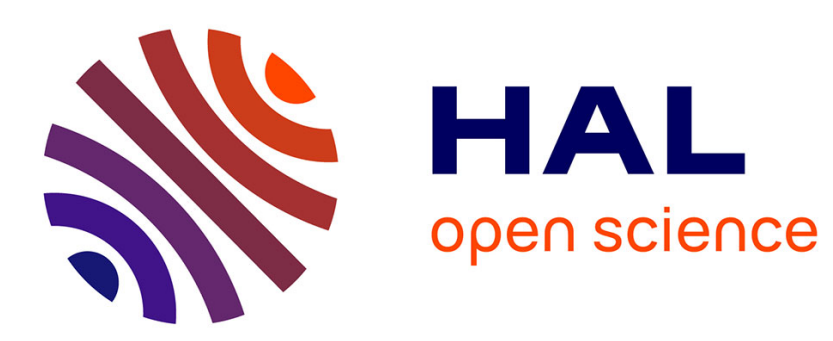

\title{
A synchronization-based state observer for impact oscillators using only collision time information
}

Michael Baumann, Remco Leine

\section{To cite this version:}

Michael Baumann, Remco Leine. A synchronization-based state observer for impact oscillators using only collision time information. International Journal of Robust and Nonlinear Control, 2015, 10.1002/rnc.3460 . hal-01326052

\section{HAL Id: hal-01326052 https://hal.science/hal-01326052}

Submitted on 6 Jun 2016

HAL is a multi-disciplinary open access archive for the deposit and dissemination of scientific research documents, whether they are published or not. The documents may come from teaching and research institutions in France or abroad, or from public or private research centers.
L'archive ouverte pluridisciplinaire HAL, est destinée au dépôt et à la diffusion de documents scientifiques de niveau recherche, publiés ou non, émanant des établissements d'enseignement et de recherche français ou étrangers, des laboratoires publics ou privés. 


\title{
A synchronization-based state observer for impact oscillators using only collision time information
}

\author{
Michael Baumann ${ }^{1}$ and Remco I. Leine ${ }^{2}$ \\ ${ }^{1}$ Institute of Mechanical Systems, ETH Zurich, Zurich, 8092, Switzerland \\ ${ }^{2}$ Institute for Nonlinear Mechanics, University of Stuttgart, Stuttgart, 70569, Germany
}

\begin{abstract}
We present the design of a state observer for Lagrangian systems subjected to frictionless geometric unilateral constraints. A master-slave synchronization setup is used in which the unidirectional coupling only consists of the information of the impact time instants. After a brief synchronization phase, the obtained observer replicates the full state of the observed system, independently of the initial conditions and even in the presence of accumulation points (Zeno behavior).

The key idea is that the (virtual) observer system is subjected to switched kinematic unilateral constraints such that it may enjoy the property of incremental stability when the impact law is maximal monotone. The main inequality impact laws for hard unilateral constraints, that is, the generalized Poisson's and Newton's impact law, are under mild assumptions maximal monotone, which is a stronger condition than dissipativity. The results are applied to two different examples of mechanical impact oscillators.
\end{abstract}

KEY WORDS: non-smooth dynamics; impact theory; measure differential inclusion; unilateral constraint; master-slave synchronization

\section{INTRODUCTION}

In this paper, we prove sufficient conditions for incremental stability of Lagrangian systems subjected to switched kinematic unilateral constraints and apply the results to the observer design of impact oscillators. The proposed observer uses only the Boolean contact information, that is, which contacts of the observed system are open or closed. No additional measurements of the state are necessary.

Incremental stability, being a system property, and several similar notions have been presented in the literature [1-5]. A system, possibly excited by an input (e.g., a forced mechanical system), is called (attractively) incrementally stable if all solution curves approach each other in forward time. Incremental stability is beneficial in many control problems such as stabilization, tracking control, output regulation problems, synchronization, and observer design [6-9].

A closely related stability notion of dynamical systems is the concept of convergence from nonlinear stability theory, which has been developed in the 1960s in the Russian research community [10-12] and is currently a topic of active research (e.g., [6]). A convergent system guarantees the existence of a globally attractively stable steady-state solution which is bounded on the whole time axis. Therefore, an incrementally stable system that admits a steady-state solution is convergent. However, incremental stability and convergence are distinct stability notions as has been shown in [13]. Although the convergence property has useful implications, the existence of a steady-state solution is no prerequisite with regard to synchronization and observer design. 
The present paper is concerned with the dynamics of Lagrangian systems with unilateral constraints, that is, mechanical systems with contacts. Such type of non-smooth systems can conveniently be described using the framework of measure differential inclusions [14-19]. Within this framework, the system states are assumed to be special functions of locally bounded variation [20]. Phenomena such as accumulation points (infinitely many jumps in a finite time interval) are not ruled out.

A classical problem in control theory is how to obtain state estimates for systems of which not the full state is measured and/or the measurements are corrupted by noise. The design of state observers for non-smooth systems is a challenging task, because classical approaches such as Kalman filtering, Luenberger observers, or high-gain observers [21-23] are not (directly) applicable. A commonly used approach is to design model-based state observers of the same dimension as the observed system such that the state of the observer acts directly as estimate. In this case, the objective becomes a synchronization problem as has been studied in, for example, [24, 25]. The link between observer design and synchronization is discussed in [26].

Observer design for various classes of non-smooth systems has been widely investigated in the literature. In [27], a convergence-based approach has been presented for a synchronization problem for piecewise affine systems, whereas observer design for piecewise linear systems is discussed in [28]. The problem of observer design for Lur'e systems with multivalued mappings in the feedback path, which can be described by differential inclusions, is discussed in [29]. For systems with impulsive motion, we need to distinguish between settings in which the states of the observer and the reference trajectory jump at the same time or not. If the impact time instants do not coincide, then the (Euclidean) observer error may exhibit a 'peaking behavior' as has been discussed in $[16,30$, 31]. In this case of mismatched discontinuities, a more sophisticated definition of an observer error is necessary as has been introduced in [32]. Otherwise, if the discontinuities occur at the same time instants, then (attractive) stability of the error dynamics can be shown if there exists a Lyapunov function using the (Euclidean) error that is non-increasing during neither continuous nor impulsive motion. In [33] and [34], state observers for impacting mechanical systems in the absence of accumulation points have been presented. The case of coinciding impact time instants has also been studied in [9] for linear complementarity systems and in [35] for measure differential inclusions. All these proposed observers rely on continuous measurements of the observed system.

The novelty of the paper is that an observer for impact oscillators is developed, which uses only the knowledge of the impact time instants and no additional continuous measurement is necessary. A key aspect in the observer design is that we introduce a conceptually new type of unilateral constraints to ensure coinciding impact time instants of the observer and the observed system. The class of Lagrangian systems subjected to unilateral constraints, which we consider here, does not require the strict passivity property, as has been assumed in, for example, [35]. More precisely, the transfer matrix of the linear part of the system is in our case (in the absence of additional measurements) only positive real and not strictly positive real [36]. Mechanical systems (without any feedback) do generally not fulfill the strict passivity condition.

The paper contributes to the research fields non-smooth dynamics, stability theory, and control theory in a number of different ways. Firstly, the concept of switched kinematic unilateral constraints is introduced in this paper. Using this concept, it can be ensured that (when comparing different solution curves) the impact time instants coincide, omitting the undesired 'peaking behavior'. Secondly, it is shown under which conditions Lagrangian systems subjected to switched kinematic unilateral constraints enjoy the property of attractive incremental stability. Finally, an observer design for Lagrangian systems with geometric unilateral constraints is presented using the attractive incremental stability property. The proposed observer is based on master-slave synchronization, where the unidirectional coupling consists only of the information of impact time instants.

This paper is organized as follows. The property of attractive incremental stability is defined for measure differential inclusions in Section 2. Various unilateral constraints for mechanical systems, especially switched kinematic unilateral constraints, are discussed in Section 3. Subsequently, Section 4 briefly reviews the generalized Newton's and generalized Poisson's impact law and discusses their compatibility with switched constraints. The property of maximal monotonicity of impact laws and its implications is discussed in Section 5. It is shown in Section 6 under which 
conditions Lagrangian systems with switched kinematic unilateral constraints are attractively incrementally stable. The application to synchronization-based observer design is presented in Section 7, which is illustrated with two examples of mechanical impact oscillators. Finally, the relationship with the concept of convergence and other conclusions are discussed in Section 8.

\section{ATTRACTIVE INCREMENTAL STABILITY}

Attractive incremental stability (a.i.s.) is a stability property of a dynamical system that implies that all solution curves approach each other and remain close in a Lyapunov sense. To be more precise, we define the attractive incremental stability property of a time-invariant measure differential inclusion with inputs of the form

$$
\mathrm{d} \boldsymbol{x} \in \mathrm{d} \boldsymbol{\Gamma}(\boldsymbol{x}, \boldsymbol{w}),
$$

where $\boldsymbol{x}(t) \in \mathbb{R}^{n}$ is the state vector and $\boldsymbol{w}(t) \in \mathbb{R}^{d}$ is the time-varying input [16]. The admissible set of the states is given by the set $\mathcal{A}$, and we will restrict the input $\boldsymbol{w}(t)$ to a certain class of functions $\mathbb{W}$.

A solution $\boldsymbol{x}(t)=\varphi\left(t, t_{0}, x_{0}\right)$ of the measure differential inclusion (1) with the initial condition $\boldsymbol{x}_{0}$ is a function $\boldsymbol{x}: \mathbb{R} \rightarrow \mathbb{R}^{n}$, being of locally bounded variation, which fulfills (1) for all $t \geqslant t_{0}$ and which is defined almost everywhere on the time axis [16]. The term almost everywhere captures that the state is not defined at its discontinuity points. In the following, almost everywhere will be referred to as a.e. and is always considered with respect to the Lebesgue measure.

\section{Definition 1}

System (1) is said to be attractively incrementally stable if for all $t_{0} \in \mathbb{R}$, admissible initial conditions $\boldsymbol{x}_{1}, \boldsymbol{x}_{2} \in \mathcal{A}$, inputs $\boldsymbol{w}(t) \in \mathbb{W}$, and corresponding solution curves $\boldsymbol{\varphi}\left(t, t_{0}, \boldsymbol{x}_{i}\right)(i=1,2)$, it holds that for any $\varepsilon>0$, there exists a $\delta=\delta(\varepsilon)$ such that $\left\|\boldsymbol{x}_{1}-\boldsymbol{x}_{2}\right\|<\delta$ implies $\| \boldsymbol{\varphi}\left(t, t_{0}, \boldsymbol{x}_{1}\right)-$ $\boldsymbol{\varphi}\left(t, t_{0}, \boldsymbol{x}_{2}\right) \|<\varepsilon$ for almost all $t \geqslant t_{0}$ and additionally $\lim _{t \rightarrow \infty}\left\|\varphi\left(t, t_{0}, x_{1}\right)-\boldsymbol{\varphi}\left(t, t_{0}, \boldsymbol{x}_{2}\right)\right\|=0$.

A measure differential inclusion of the form (1) is therefore a.i.s. if all solution curves are globally uniformly attractively stable. An a.i.s. system 'forgets' the initial conditions, and all solutions converge to each other (Figure 1). The wording 'attractively stable' has been used instead of the usual term 'asymptotically stable', because attractivity of solutions of (measure) differential inclusions can be asymptotic or symptotic (finite-time attractivity) [16]. Definition 1 of incremental stability considers only global attractivity and uniform stability. Other incremental stability notions have been presented in literature (e.g., $[4,5])$.

\section{Remark 1}

Closely related to incremental stability is (uniform) convergence [10, 12]. A uniformly convergent system admits a globally uniformly attractively stable steady-state solution, which is bounded for almost all $t \in \mathbb{R}$. If an a.i.s. system admits a steady-state solution, then the system is uniformly convergent $[13,16]$. A standard way to prove the existence of a steady-state solution is to find a compact positively invariant set. This type of proof has been given in the literature [12,37] for certain classes of smooth dynamical systems, and the extension of this technique to non-smooth systems described by measure differential inclusions has been proposed in [16]. A uniformly convergent system has useful properties such as uniqueness of the steady-state solution and that a periodic (or constant) input implies a periodic (or constant) steady-state solution. These properties have been proven in [6]

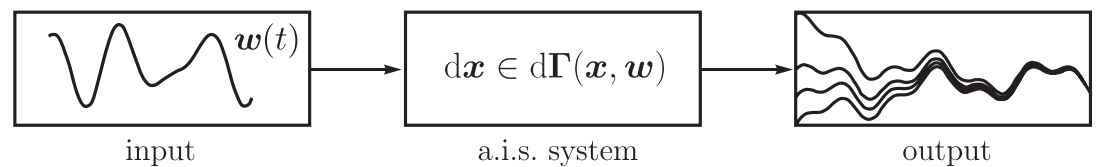

Figure 1. All solutions of an attractively incrementally stable (a.i.s.) system converge to one another for every input $w(t) \in \mathbb{W}$. 
for differential inclusions. Here, we develop a synchronization-based state observer for mechanical systems with impacts, and the existence of a steady-state solution is therefore not essential. Hence, we focus on the property of attractive incremental stability.

\section{MECHANICAL SYSTEMS WITH UNILATERAL CONSTRAINTS}

We consider an $f$-DOF linear time-invariant Lagrangian system. The system is subjected to a time-dependent external forcing and perfect unilateral constraints. Let $\boldsymbol{q} \in \mathbb{R}^{f}$ be the generalized coordinates and $\boldsymbol{u} \in \mathbb{R}^{f}$ be the generalized velocities. The non-impulsive dynamics of the system is described by the kinematic equation $\dot{\boldsymbol{q}}=\boldsymbol{u}$ a.e. and the equation of motion

$$
\boldsymbol{M} \dot{\boldsymbol{u}}+\boldsymbol{C u}+\boldsymbol{K} \boldsymbol{q}=\boldsymbol{W} \boldsymbol{\lambda}+\boldsymbol{f}(t) \text { a.e., }
$$

where the mass matrix $\boldsymbol{M} \in \mathbb{R}^{f \times f}$ and the stiffness matrix $\boldsymbol{K} \in \mathbb{R}^{f \times f}$ are symmetric. The system matrices $\boldsymbol{M}, \boldsymbol{C}$, and $\boldsymbol{K}$ are time invariant and positive definite. The time-dependent external forcing $\boldsymbol{f}(t)$ is bounded. The motion of the system is restricted by $m$ unilateral constraints $0 \leqslant \boldsymbol{g}(\boldsymbol{q}) \in$ $\mathbb{R}^{m}$, which invoke constraint forces $\lambda \in \mathbb{R}^{m}$ together with generalized force directions $\boldsymbol{W} \in \mathbb{R}^{f \times m}$, which we assume to be linearly independent.

Correspondingly, the impulsive dynamics is described by the impact equation

$$
\boldsymbol{M}\left(\boldsymbol{u}^{+}-\boldsymbol{u}^{-}\right)=\boldsymbol{W} \boldsymbol{\Lambda},
$$

where $\boldsymbol{u}^{-}$and $\boldsymbol{u}^{+}$denote the pre-impact and post-impact velocities and $\boldsymbol{\Lambda} \in \mathbb{R}^{m}$ are the impulsive constraint forces. The generalized coordinates $\boldsymbol{q}(t)$ are absolutely continuous w.r.t. time, whereas the generalized velocities $\boldsymbol{u}(t)$ are special functions of locally bounded variation.

The equation of motion (2) and the impact (3) need to be complemented with a force law for the constraint forces $\lambda$ and an impact law for the constraint impulses $\boldsymbol{\Lambda}$. These constitutive laws depend on the type of the unilateral constraints and typically operate on local kinematic quantities. We will assume that the constraint distances $\boldsymbol{g}(\boldsymbol{q})$ are an affine function of the generalized coordinates $\boldsymbol{q}$, and the constraint velocities $\boldsymbol{\gamma}(\boldsymbol{u})$ are a linear function of the generalized velocities $\boldsymbol{u}$, that is,

$$
\begin{aligned}
& \boldsymbol{g}=\boldsymbol{W}^{\mathrm{T}} \boldsymbol{q}+\boldsymbol{g}_{r}, \\
& \boldsymbol{\gamma}=\boldsymbol{W}^{\mathrm{T}} \boldsymbol{u},
\end{aligned}
$$

where $\boldsymbol{g}_{r} \in \mathbb{R}^{m}$ is a constant vector. In the following, the force and impact laws for the unilateral constraints will be formulated using the so-called unilateral primitive Upr [19] defined by

$$
\mathrm{Upr}(x)=\partial \Psi_{\mathbb{R}_{0}^{+}}= \begin{cases}0 & \text { if } x>0 \\ (-\infty, 0] & \text { if } x=0, \\ \emptyset & \text { if } x<0\end{cases}
$$

which is the subdifferential of the indicator function $\Psi_{\mathbb{R}_{0}^{+}}$on the set $\mathbb{R}_{0}^{+}$. Using the unilateral primitive Upr, an inequality complementarity is expressed as $-y \in \operatorname{Upr}(x)$, which is identical to $x \geqslant 0$, $y \geqslant 0$, and $x y=0$. If $\boldsymbol{x}, \boldsymbol{y} \in \mathbb{R}^{n}$, we use the notation $-\boldsymbol{y} \in \operatorname{Upr}(\boldsymbol{x}) \Longleftrightarrow \boldsymbol{0} \leqslant \boldsymbol{x} \perp \boldsymbol{y} \geqslant \mathbf{0}[16,19]$.

Three types of perfect unilateral constraints will be considered:

(1) A kinematic unilateral constraint restricts the sign of a constraint velocity $\gamma_{i}(\boldsymbol{u}) \geqslant 0$ (where $i$ is the index of the considered unilateral constraint), thereby allowing for relative motion in positive direction and blocking in the opposite direction. Kinematic unilateral constraints are also known as one-way clutches or sprag clutches. The force law of a kinematic unilateral constraint is described by the inequality complementarity

$$
-\lambda_{i} \in \operatorname{Upr}\left(\gamma_{i}\right) \text {. }
$$


(2) A switched kinematic unilateral constraint imposes a kinematic unilateral constraint $\gamma_{i}(\boldsymbol{u}) \geqslant 0$ whenever the corresponding Boolean switching function $\chi_{i}(t)=1$ and imposes no constraint if $\chi_{i}(t)=0$. The switching function $\chi_{i}(t): \mathbb{R} \mapsto\{0,1\}$ is an external input and thus independent of the states. The force law is described by the inequality complementarity

$$
-\lambda_{i} \in \begin{cases}\operatorname{Upr}\left(\gamma_{i}\right) & \text { if } \chi_{i}(t)=1, \\ 0 & \text { if } \chi_{i}(t)=0 .\end{cases}
$$

(3) A geometric unilateral constraint, also known as impenetrability constraint, restricts the sign of a constraint distance $g_{i}(\boldsymbol{q}) \geqslant 0$. Its force law is described by the inequality complementarity (also referred to as Signorini's law)

$$
-\lambda_{i} \in \operatorname{Upr}\left(g_{i}\right)
$$

which can also be written on velocity level [19] as

$$
-\lambda_{i} \in \begin{cases}\operatorname{Upr}\left(\gamma_{i}\right) & \text { if } g_{i}(\boldsymbol{q})=0, \\ 0 & \text { if } g_{i}(\boldsymbol{q})>0 .\end{cases}
$$

The switched kinematic unilateral constraints are 'time triggered', and $\chi(t)$ is an external input independent of the constraint velocities $\gamma$. This is contrary to the 'state-triggered' geometric unilateral constraints, where the set of closed contacts is given by the constraint distances $g$. In the case of geometric unilateral constraints, there exists a causality between the constraint distances $\boldsymbol{g}$ and the constraint velocities $\boldsymbol{\gamma}$ given by $\boldsymbol{\gamma}=\dot{\boldsymbol{g}}$ for almost all $t$. In order to relate these two types of constraints, we make the following definition.

\section{Definition 2}

The Boolean switching functions $\chi(t)$ are called to be generated by $\boldsymbol{g}(t)$ if every component $\chi_{i}(t)$ of $\chi(t)$ fulfills

$$
\chi_{i}(t)= \begin{cases}1 & \text { if } g_{i}(t)=0, \\ 0 & \text { if } g_{i}(t)>0 .\end{cases}
$$

By comparing the force laws (7) and (9), we observe that geometric unilateral constraints are equivalent to switched kinematic unilateral constraints if the Boolean switching functions $\chi(t)$ are generated by the constraint distances $g$.

\section{IMPACT LAWS FOR PERFECT UNILATERAL CONSTRAINTS}

In this section, we briefly summarize the generalized Newton's impact law and the generalized Poisson's impact law, being the main inequality impact laws for hard unilateral constraints $[16,19$, 38]. Each of these impact laws will be formulated here for perfect (frictionless) unilateral constraints but may very well be extended to unilateral constraints with friction.

\subsection{Generalized Newton's impact law}

The classical Newton's impact law for a closed geometric unilateral constraint $i$

$$
\gamma_{i}^{+}=-\varepsilon_{i} \gamma_{i}^{-}, \quad g_{i}=0, \quad 0 \leqslant \varepsilon_{i} \leqslant 1
$$

relates the post-impact velocity $\gamma_{i}^{+}$to the pre-impact velocity $\gamma_{i}^{-}$by a Newtonian coefficient of restitution $\varepsilon_{i} \in[0,1]$. The case $\varepsilon_{i}=1$ corresponds to a completely elastic impact, whereas $\varepsilon_{i}=0$ corresponds to a completely inelastic impact. The impact causing the sudden change in constraint velocity is accompanied by a constraint impulse $\Lambda_{i}>0$. A unilateral constraint is called superfluous if it does not participate in the impact, although the geometric unilateral constraint is closed/active $\left(g_{i}=0\right.$ for a geometric unilateral constraint or $\chi_{i}=1$ for a switched unilateral constraint). 
For $\gamma_{i}^{-}<0$, the occurrence of such a superfluous constraint only occurs for multi-constraint situations. Following [14, 38], we generalize the classical Newton's impact law to account for superfluous constraints by allowing post-impact constraint velocities larger than those prescribed by Newton's impact law in the case of a non-vanishing impulse, that is, $\gamma_{i}^{+} \geqslant-\varepsilon_{i} \gamma_{i}^{-}$. Summarizing, two cases can occur at a closed/active unilateral constraint $i$ :

(1) The unilateral constraint is participating in the impact process, that is, $\Lambda_{i}>0$ and $\gamma_{i}^{+}=$ $-\varepsilon_{i} \gamma_{i}^{-}$.

(2) The unilateral constraint is superfluous, that is, $\Lambda_{i}=0$ and $\gamma_{i}^{+} \geqslant-\varepsilon_{i} \gamma_{i}^{-}$.

These two cases are combined in an inequality complementarity impact law on velocity-impulse level as $0 \leqslant \Lambda_{i} \perp\left(\gamma_{i}^{+}+\varepsilon_{i} \gamma_{i}^{-}\right) \geqslant 0$, whereas $\Lambda_{i}=0$ if $g_{i}>0$ (or $\chi_{i}=0$ ). Using the unilateral primitive and $\xi_{i}=\gamma_{i}^{+}+\varepsilon_{i} \gamma_{i}^{-}$, the inequality complementarity can be written in the form

$$
-\Lambda_{i} \in \begin{cases}\mathrm{Upr}\left(\xi_{i}\right) & \text { if } g_{i}=0 \\ 0 & \text { if } g_{i}>0\end{cases}
$$

The generalized Newton's impact law (12) is the simplest inequality impact law for hard unilateral constraints as it only uses a single inequality complementarity. If the pre-impact velocities fulfill $\gamma_{i}^{-} \leqslant 0$, then the post-impact velocities are admissible because of $\gamma_{i}^{+} \geqslant-\varepsilon_{i} \gamma_{i}^{-} \geqslant 0$. The kinematic consistency [39] of the post-impact velocities $\gamma_{i}^{+} \geqslant 0$ of the generalized Newton's impact law therefore follows from the sign of the pre-impact velocities. This sign condition naturally holds for geometric unilateral constraints as the bodies have to approach each other in order to come into contact. However, this condition does no longer hold for switched kinematic unilateral constraints, and the post-impact velocities are not generally admissible. The generalized Newton's impact law (12) is therefore not compatible with switched kinematic unilateral constraints.

\subsection{Generalized Poisson's impact law}

The generalized Poisson's impact law [38, 40] distinguishes between a compression phase and an expansion phase. The impulsive forces maximize the reduction in the associated kinetic energy within their admissible set. The compression phase ends when the normal relative velocities vanish. This corresponds to a completely inelastic collision and can be represented by the generalized Newton's impact law with $\varepsilon_{i}=0$ as

$$
\boldsymbol{M}\left(\boldsymbol{u}^{C}-\boldsymbol{u}^{-}\right)=\boldsymbol{W} \boldsymbol{\Lambda}^{C}, \quad-\Lambda_{i}^{C} \in \operatorname{Upr}\left(\gamma_{i}^{C}\right) .
$$

The compression constraint impulses $\Lambda^{C}$ and the constraint velocities after the compression phase $\boldsymbol{\gamma}^{C}$ are therefore nonnegative (by components).

The deformation energy gained during the compression is partly released during the expansion phase and reconverted into kinetic energy. The dissipative behavior is expressed by the Poisson's restitution coefficients $\varepsilon_{i}$, which relate the expansion impulse to the compression impulse. The expansion phase is described by an inequality complementarity as

$$
\boldsymbol{M}\left(\boldsymbol{u}^{+}-\boldsymbol{u}^{C}\right)=\boldsymbol{W} \boldsymbol{\Lambda}^{E}, \quad-\left(\Lambda_{i}^{E}-\varepsilon_{i} \Lambda_{i}^{C}\right) \in \operatorname{Upr}\left(\gamma_{i}^{+}\right) .
$$

Addition of (13) and (14) yields (3) with $\Lambda=\Lambda^{C}+\Lambda^{E}$. The generalized Poisson's impact law is able to describe certain restitution effects of multi-constraint collisions that are not possible to describe with the generalized Newton's impact law. The differences between the generalized Newton's impact law and the generalized Poisson's impact law are explained in detail in [38-40].

The inequality complementarity of the expansion phase explicitly guarantees the kinematic consistency of the post-impact velocities $\gamma_{i}^{+} \geqslant 0$ (independent of the sign of the pre-impact velocities). This is achieved by increasing the expansion constraint impulse $\Lambda_{i}^{E}$ for negative $\gamma_{i}^{+}$ until admissible post-impact velocities are achieved. Therefore, the generalized Poisson's impact law is, in contrast to the generalized Newton's impact law, compatible with switched kinematic unilateral constraints. 


\section{MAXIMAL MONOTONICITY OF IMPACT LAWS}

An impact law is usually expressed using the pre-impact and post-impact velocities $\boldsymbol{\gamma}^{-}$and $\boldsymbol{\gamma}^{+}$ (or $\boldsymbol{u}^{-}$and $\boldsymbol{u}^{+}$). In [17, 41, 42], a different kind of representation is proposed, which allows reflecting the specific mathematical properties of the impact law. The key idea is to express the impact law in dual variables. Hereto, we consider the kinetic energy $T$ dissipated in the impact process

$$
\begin{aligned}
T^{+}-T^{-} & =\frac{1}{2} \boldsymbol{u}^{+\mathrm{T}} \boldsymbol{M} \boldsymbol{u}^{+}-\frac{1}{2} \boldsymbol{u}^{-\mathrm{T}} \boldsymbol{M} \boldsymbol{u}^{-}=\frac{1}{2}\left(\boldsymbol{u}^{+}+\boldsymbol{u}^{-}\right)^{\mathrm{T}} \boldsymbol{M}\left(\boldsymbol{u}^{+}-\boldsymbol{u}^{-}\right) \\
& =\frac{1}{2}\left(\boldsymbol{u}^{+}+\boldsymbol{u}^{-}\right)^{\mathrm{T}} \boldsymbol{W} \boldsymbol{\Lambda}=\frac{1}{2}\left(\boldsymbol{\gamma}^{+}+\boldsymbol{\gamma}^{-}\right)^{\mathrm{T}} \boldsymbol{\Lambda}=\overline{\boldsymbol{\gamma}}^{\mathrm{T}} \boldsymbol{\Lambda},
\end{aligned}
$$

where the impact (3), the kinematic relationship (4), and the symmetry of the mass matrix have been used. In (15), we identify the kinematic quantity

$$
\bar{\gamma}=\frac{1}{2}\left(\gamma^{+}+\gamma^{-}\right)
$$

which one could call the 'mean' constraint velocity of the impact. The kinematic variable $\bar{\gamma}$ and the kinetic variable $\boldsymbol{\Lambda}$ are therefore dual variables in the sense that $\delta \overline{\boldsymbol{\gamma}}^{\mathrm{T}} \boldsymbol{\Lambda}$ is the virtual work of the impulsive constraint force. Hence, we formulate the impact law as a set-valued relationship between these dual variables as

$$
-\Lambda \in \mathcal{H}(\bar{\gamma}) .
$$

The set-valued impact map $\mathcal{H}: \mathbb{R}^{m} \rightrightarrows \mathbb{R}^{m}$ depends on which contacts are closed or open.

It will become apparent in Section 6 that the maximal monotonicity of the impact map $\mathcal{H}$ is essential for the attractive incremental stability of Lagrangian systems with unilateral constraints. We first recall the definition of maximal monotonicity of a set-valued function [43].

Definition 3 (Maximal monotonicity of a set-valued function)

A set-valued function $\mathcal{F}$ is called monotone if its graph is monotone in the sense that

$$
\left(y_{1}-y_{2}\right)^{\mathrm{T}}\left(x_{1}-x_{2}\right) \geqslant 0
$$

for all $\left(x_{1}, y_{1}\right)$ and $\left(x_{2}, y_{2}\right)$ such that $y_{1} \in \mathcal{F}\left(x_{1}\right)$ and $y_{2} \in \mathcal{F}\left(x_{2}\right)$. Furthermore, $\mathcal{F}$ is called maximal monotone if it is monotone and if there exists no other monotone set-valued function whose graph strictly contains the graph of $\mathcal{F}$.

The generalized Newton's impact law and the generalized Poisson's impact law are both maximal monotone for a global coefficient of restitution. This property persists if the coefficients of restitutions are different but close to each other or sufficiently small [41, 42].

\section{Remark 2}

Typically, an impact law fulfills that vanishing pre-impact velocities $\boldsymbol{\gamma}^{-}=\mathbf{0}$ lead to vanishing impulsive forces $\boldsymbol{\Lambda}$ and therefore vanishing post-impact velocities $\boldsymbol{\gamma}^{+}=\boldsymbol{\gamma}^{-}=\mathbf{0}$. This implies the natural condition $\mathbf{0} \in \mathcal{H}(\mathbf{0})$. If the impact map is maximal monotone and the natural condition is fulfilled, we obtain $-(\boldsymbol{\Lambda}-\mathbf{0})^{\mathrm{T}}(\bar{\gamma}-\mathbf{0})=-\left(T^{+}-T^{-}\right) \geqslant 0$. In this case, the impact dissipation is positive. That is, the impact work is negative. We conclude that maximal monotonicity of the impact law is a stronger condition than dissipativity. The generalized Newton's impact law and the generalized Poisson's impact law are both dissipative for perfect unilateral constraints, $\varepsilon_{i} \in[0,1]$ and $\boldsymbol{\gamma}_{i}^{-} \leqslant 0[39,40]$.

\section{Remark 3}

If the impact map $\mathcal{H}$ enjoys the cyclic maximal monotonicity property, which is an even stronger condition than maximal monotonicity [43], then the impact map can be written as the subdifferential of a convex proper lower semi-continuous dissipation function as is discussed in [41]. 
The impact law can also be defined by the mapping $S: \boldsymbol{\gamma}^{+}=S\left(\gamma^{-}\right)$from pre-impact to postimpact constraint velocities or the mapping $Z: \boldsymbol{u}^{+}=Z\left(\boldsymbol{u}^{-}\right)$from pre-impact to post-impact generalized velocities. In [41], the impact map $\mathcal{H}$ and the mappings $S$ and $Z$ are explicitly given for the generalized Newton's impact map. The property of maximal monotonicity of $\mathcal{H}$ is related to the non-expansivity of the mappings $S$ and $Z$ in the following way.

\section{Proposition 1}

If and only if $\mathcal{H}$ is maximal monotone, then the mappings $S$ and $Z$ are maximal non-expansive, each in their own metric $[41,43]$, that is,

$$
\begin{array}{lll}
\boldsymbol{\gamma}^{+}=S\left(\boldsymbol{\gamma}^{-}\right) & \text {maximal non-expansive in } \boldsymbol{G}^{-1}: & \left\|\boldsymbol{\gamma}_{1}^{+}-\boldsymbol{\gamma}_{2}^{+}\right\|_{\boldsymbol{G}^{-1}}^{2} \leqslant\left\|\boldsymbol{\gamma}_{1}^{-}-\boldsymbol{\gamma}_{2}^{-}\right\|_{\boldsymbol{G}^{-1}}^{2}, \\
\boldsymbol{u}^{+}=Z\left(\boldsymbol{u}^{-}\right) & \text {maximal non-expansive in } \boldsymbol{M}: & \left\|\boldsymbol{u}_{1}^{+}-\boldsymbol{u}_{2}^{+}\right\|_{\boldsymbol{M}}^{2} \leqslant\left\|\boldsymbol{u}_{1}^{-}-\boldsymbol{u}_{2}^{-}\right\|_{\boldsymbol{M}}^{2},
\end{array}
$$

where $\boldsymbol{G}=\boldsymbol{W}^{\mathrm{T}} \boldsymbol{M}^{-1} \boldsymbol{W}$ is the Delassus matrix.

Proof

The monotonicity condition (18) of $\mathcal{H}$ can be written as

$$
\begin{aligned}
0 & \geqslant 2\left(\bar{\gamma}_{1}-\bar{\gamma}_{2}\right)^{\mathrm{T}}\left(\boldsymbol{\Lambda}_{1}-\boldsymbol{\Lambda}_{2}\right) \\
& =\left(\boldsymbol{\gamma}_{1}^{+}+\boldsymbol{\gamma}_{1}^{-}-\boldsymbol{\gamma}_{2}^{+}-\boldsymbol{\gamma}_{2}^{-}\right)^{\mathrm{T}} \boldsymbol{G}^{-1}\left(\boldsymbol{\gamma}_{1}^{+}-\boldsymbol{\gamma}_{1}^{-}-\boldsymbol{\gamma}_{2}^{+}+\boldsymbol{\gamma}_{2}^{-}\right) \\
& =\left\|\boldsymbol{\gamma}_{1}^{+}-\boldsymbol{\gamma}_{2}^{+}\right\|_{\boldsymbol{G}^{-1}}^{2}-\left\|\boldsymbol{\gamma}_{1}^{-}-\boldsymbol{\gamma}_{2}^{-}\right\|_{\boldsymbol{G}^{-1}}^{2},
\end{aligned}
$$

where (3), (16), and the symmetry of $\boldsymbol{G}$ have been used. Analogously, the monotonicity condition (18) of $\mathcal{H}$ can be rewritten as

$$
\begin{aligned}
0 & \geqslant 2\left(\bar{\gamma}_{1}-\bar{\gamma}_{2}\right)^{\mathrm{T}}\left(\boldsymbol{\Lambda}_{1}-\boldsymbol{\Lambda}_{2}\right) \\
& =\left(\boldsymbol{u}_{1}^{+}+\boldsymbol{u}_{1}^{-}-\boldsymbol{u}_{2}^{+}-\boldsymbol{u}_{2}^{-}\right)^{\mathrm{T}} \boldsymbol{W}\left(\boldsymbol{\Lambda}_{1}-\boldsymbol{\Lambda}_{2}\right) \\
& =\left\|\boldsymbol{u}_{1}^{+}-\boldsymbol{u}_{2}^{+}\right\|_{\boldsymbol{M}}^{2}-\left\|\boldsymbol{u}_{1}^{-}-\boldsymbol{u}_{2}^{-}\right\|_{\boldsymbol{M}}^{2},
\end{aligned}
$$

where (3), (4), (16), and the symmetry of $\boldsymbol{M}$ have been used.

The impact maps depend on the set of closed contacts, given by the switching functions $\chi(t)$ or the constraint distances $g$, which is indicated using a subscript for

$$
\begin{array}{ll}
\text { switched kinematic unilateral constraints: } & -\boldsymbol{\Lambda} \in \mathcal{H}_{\chi(t)}(\bar{\gamma}), \\
\text { and geometric unilateral constraints: } & -\boldsymbol{\Lambda} \in \mathcal{H}_{g}(\bar{\gamma}) .
\end{array}
$$

Naturally, the constraint impulses vanish if all constraints are open or switched off, respectively. If we consider two different impact processes $\left(\boldsymbol{\Lambda}_{1}, \bar{\gamma}_{1}\right)$ and $\left(\boldsymbol{\Lambda}_{2}, \bar{\gamma}_{2}\right)$ of a system with geometric unilateral constraints, then the constraint distances $g_{1}$ and $g_{2}$ are generally unequal, and therefore, the set of closed contacts differ. In this case, it is meaningless to evaluate the maximal monotonicity condition (18), because the impacting processes are described by different impact maps $\mathcal{H}_{g_{1}}$ and $\mathcal{H}_{g_{2}}$. This problem does not occur for switched kinematic unilateral constraints as the switching functions $\chi(t)$ are an external input and therefore identical for all considered solutions. Hence, for the maximal monotonicity condition (18) for switched constraints, we evaluate the condition

$$
\left(\boldsymbol{\Lambda}_{1}-\boldsymbol{\Lambda}_{2}\right)^{\mathrm{T}}\left(\bar{\gamma}_{1}-\bar{\gamma}_{2}\right) \leqslant 0
$$

with $-\boldsymbol{\Lambda}_{1} \in \mathcal{H}_{\chi(t)}\left(\bar{\gamma}_{1}\right)$ and $-\boldsymbol{\Lambda}_{2} \in \mathcal{H}_{\chi(t)}\left(\bar{\gamma}_{2}\right)$ for the same values of $\chi(t)$. The concept of switched kinematic unilateral constraints is thus necessary to exploit the maximal monotonicity property of the impact law or, equivalently, the non-expansivity of the mapping $Z$. These properties will directly be used to show the attractive incremental stability of constrained Lagrangian systems in Section 6. 


\section{ATTRACTIVE INCREMENTAL STABILITY OF LAGRANGIAN SYSTEMS WITH SWITCHED KINEMATIC UNILATERAL CONSTRAINTS}

In this section, we show how the property of maximal monotonicity leads to attractive incremental stability of constrained dynamical systems. More specifically, we consider forced linear timeinvariant Lagrangian systems with positive definite system matrices that are subjected to switched kinematic unilateral constraints. Let us briefly summarize the full set of equations. The dynamics is described by the kinematic equation, equation of motion, and impact equation

$$
\begin{aligned}
& \dot{\boldsymbol{q}}=\boldsymbol{u} \text { a.e., } \\
& \boldsymbol{M} \dot{\boldsymbol{u}}+\boldsymbol{C u}+\boldsymbol{K} \boldsymbol{q}=\boldsymbol{W} \boldsymbol{\lambda}+\boldsymbol{f}(t) \quad \text { a.e., } \\
& \boldsymbol{M}\left(\boldsymbol{u}^{+}-\boldsymbol{u}^{-}\right)=\boldsymbol{W} \boldsymbol{\Lambda}
\end{aligned}
$$

and the force and impact law

$$
\begin{aligned}
& -\lambda_{i} \in \begin{cases}\operatorname{Upr}\left(\gamma_{i}\right) & \text { if } \chi_{i}(t)=1, \\
0 & \text { if } \chi_{i}(t)=0,\end{cases} \\
& -\boldsymbol{\Lambda} \in \mathcal{H}_{\chi(t)}(\bar{\gamma}), \text { where } \bar{\gamma}=\frac{1}{2}\left(\boldsymbol{\gamma}^{+}+\boldsymbol{\gamma}^{-}\right),
\end{aligned}
$$

together with the local kinematics

$$
\boldsymbol{\gamma}=\boldsymbol{W}^{\mathrm{T}} \boldsymbol{u} .
$$

Each switched kinematic unilateral constraint $i$ is defined by a switched inequality complementarity (26) with a corresponding impact law (27) defined using the dual variables $\bar{\gamma}$ and $\boldsymbol{\Lambda}$. The damping matrix $\boldsymbol{C} \succ \mathbf{0}$ is assumed to be positive definite. The mass matrix $\boldsymbol{M}=\boldsymbol{M}^{\mathrm{T}} \succ \mathbf{0}$ as well as the stiffness matrix $\boldsymbol{K}=\boldsymbol{K}^{\mathrm{T}} \succ \boldsymbol{0}$ are assumed to be symmetric and positive definite. The Delassus matrix $\boldsymbol{G}=\boldsymbol{W}^{\mathrm{T}} \boldsymbol{M}^{-1} \boldsymbol{W} \succ \mathbf{0}$ is positive definite because of the linear independence of the generalized force directions. The constrained Lagrangian system (24)-(28) is chosen as simple as possible in order to keep the further analysis concise.

Before we state Theorem 1, which claims the a.i.s. of (24)-(28), we need to make several assumptions. One important assumption is that no switched unilateral constraint can stay persistent forever in forward time. Hereto, using the notation

$$
\tilde{a}_{\Delta t}(t)=\frac{1}{\Delta t} \int_{t}^{t+\Delta t} a(\tau) \mathrm{d} \tau \text { for a given } \Delta t>0,
$$

we define the classes of functions $\mathbb{K}$ and $\mathbb{K}^{m}$ as follows.

\section{Definition 4}

A Boolean switching function $\chi(t)$ is of class $\mathbb{K}$ if for each $t$, there exists a $t^{*}(t)>t$ and a number $\Delta t>0$ independent of $t$ such that $\tilde{\chi}_{\Delta t}\left(t^{*}\right)=0$. Furthermore, the functions $\chi(t)$ are of class $\mathbb{K}^{m}$ if each component $\chi_{i}(t)$ is of class $\mathbb{K}$.

We will make the following assumptions on the system:

(A1) The switching functions $\chi(t)$ are of class $\mathbb{K}^{m}$ and are generated by absolutely continuous functions.

(A2) The external forcing $\boldsymbol{f}(t)$ is bounded, that is, $\sup _{t \in \mathbb{R}}\|\boldsymbol{f}(t)\| \leqslant f_{\max }$, for a given bound $f_{\max }<\infty$.

(A3) The impact map $\mathcal{H}_{\chi(t)}(\bar{\gamma})$ is maximal monotone. 
Assumption (A1) guarantees that the Lebesgue measure of the sum of time intervals for which each constraint is switched off is infinite. This assumption does not exclude accumulation points, but it guarantees that eventually, every constraint will open again after an accumulation point and subsequent phase of persistent contact. Furthermore, the switching functions $\chi(t)$ are assumed to be generated by absolutely continuous functions from which follows that all intervals during which a constraint is switched off are open time intervals. This property together with Assumption (A2) is used for the existence of solutions, but this is not in the scope of this paper.

The system (24)-(28) with given switching functions $\chi(t)$ can be written in the form (1), where the input $\boldsymbol{w}(t)$ comprises the switching functions $\chi(t)$ and external forcing $\boldsymbol{f}(t)$. Now, we are ready to make the desired statement concerning the a.i.s. of (24)-(28) in the following theorem.

\section{Theorem 1}

System (24)-(28) for given switching functions $\chi(t)$ together with Assumptions (A1)-(A3) is attractively incrementally stable.

Proof

According to Definition 1, we need to show that all solution curves of system (24)-(28) are globally uniformly attractively stable. Therefore, consider two arbitrary solutions $\left(\boldsymbol{q}_{1}(t), \boldsymbol{u}_{1}(t)\right)$ and $\left(\boldsymbol{q}_{2}(t), \boldsymbol{u}_{2}(t)\right)$ of system (24)-(28) for given switching functions $\chi(t)$. Let $\boldsymbol{e}=\boldsymbol{q}_{1}-\boldsymbol{q}_{2}$ and $\boldsymbol{v}=\boldsymbol{u}_{1}-\boldsymbol{u}_{2}$ be the position and the velocity error between these two solutions, which we gather in the state vector $\boldsymbol{x}_{e}=\left(\boldsymbol{e}^{\mathrm{T}} \boldsymbol{v}^{\mathrm{T}}\right)^{\mathrm{T}}$ of the error dynamics. The Lyapunov function

$$
V\left(\boldsymbol{x}_{e}\right)=\frac{1}{2}\left\|\boldsymbol{u}_{1}-\boldsymbol{u}_{2}\right\|_{\boldsymbol{M}}^{2}+\frac{1}{2}\left\|\boldsymbol{q}_{1}-\boldsymbol{q}_{2}\right\|_{\boldsymbol{K}}^{2}=\frac{1}{2}\|\boldsymbol{v}\|_{\boldsymbol{M}}^{2}+\frac{1}{2}\|\boldsymbol{e}\|_{\boldsymbol{K}}^{2}
$$

gives a notion of distance between these two solutions and is positive definite because of the positive definiteness of the matrices $\boldsymbol{M}$ and $\boldsymbol{K}$. The two solutions agree if and only if $V=0$.

The Lyapunov function $V\left(\boldsymbol{x}_{e}(t)\right)$ given by (30) evaluated along the solution $\boldsymbol{x}_{e}(t)$ is a special function of locally bounded variation in $t$, because the generalized velocities $\boldsymbol{u}(t)$ are special functions of bounded variation and the generalized coordinates $\boldsymbol{q}(t)$ are absolutely continuous. Therefore, the differential measure of the Lyapunov function $\mathrm{d} V$ contains a density $\dot{V}$ with respect to the Lebesgue measure $\mathrm{d} t$ and a density $V^{+}-V^{-}$with respect to a purely atomic measure $\mathrm{d} \eta$ [20]. Because of the quadratic form of $V$, we have $\mathrm{d} V=\dot{V} \mathrm{~d} t+\left(V^{+}-V^{-}\right) \mathrm{d} \eta=\frac{1}{2}\left(\boldsymbol{v}^{+}+\boldsymbol{v}^{-}\right)^{\mathrm{T}} \boldsymbol{M} \mathrm{d} \boldsymbol{v}+$ $\boldsymbol{e}^{\mathrm{T}} \boldsymbol{K} \mathrm{d} \boldsymbol{e}$. The density $\dot{V}$ is given by

$$
\begin{aligned}
\dot{V}\left(t, \boldsymbol{x}_{e}\right) & =\boldsymbol{v}^{\mathrm{T}} \boldsymbol{M} \dot{\boldsymbol{v}}+\boldsymbol{e}^{\mathrm{T}} \boldsymbol{K} \dot{\boldsymbol{e}} \\
& =\boldsymbol{v}^{\mathrm{T}}\left(-\boldsymbol{C} \boldsymbol{v}+\boldsymbol{W}\left(\lambda_{1}-\lambda_{2}\right)\right) \\
& =-\|\boldsymbol{v}\|_{\overline{\boldsymbol{C}}}^{2}+\left(\boldsymbol{\gamma}_{1}-\boldsymbol{\gamma}_{2}\right)^{\mathrm{T}}\left(\lambda_{1}-\lambda_{2}\right),
\end{aligned}
$$

where $\overline{\boldsymbol{C}}=\frac{1}{2}\left(\boldsymbol{C}+\boldsymbol{C}^{\mathrm{T}}\right)$ denotes the symmetric part of $\boldsymbol{C}$. Hence, the positive definiteness of $\boldsymbol{C}$ (and thus of $\boldsymbol{C}$ ) and the maximal monotonicity of the force law (26) imply $\dot{V}\left(t, \boldsymbol{x}_{e}\right) \leqslant-W\left(\boldsymbol{x}_{e}\right)$ a.e., where $W\left(\boldsymbol{x}_{e}\right)=\left\|\boldsymbol{u}_{1}-\boldsymbol{u}_{2}\right\|_{\overline{\boldsymbol{C}}}^{2}$ is a positive semi-definite function in $\boldsymbol{x}_{e}$.

The jump in the Lyapunov function at impulsive time instants is given by

$$
V^{+}-V^{-}=\frac{1}{2}\left(\boldsymbol{v}^{+}+\boldsymbol{v}^{-}\right)^{\mathrm{T}} \boldsymbol{M}\left(\boldsymbol{v}^{+}-\boldsymbol{v}^{-}\right)=\frac{1}{2}\left\|\boldsymbol{v}^{+}\right\|_{M}^{2}-\frac{1}{2}\left\|\boldsymbol{v}^{-}\right\|_{M}^{2} .
$$

The impact map $\mathcal{H}_{\chi(t)}(\bar{\gamma})$ is maximal monotone by Assumption (A3), which implies nonexpansivity of the mapping $Z$ according to Proposition 1. The non-expansivity condition (20) directly implies that the density (32) is non-positive and, consequently, the Lyapunov function $V$ cannot increase during neither continuous nor discontinuous flow. Therefore, the equilibrium $\boldsymbol{x}_{e}=\mathbf{0}$ is uniformly stable [16]. Furthermore, because the Lyapunov function $V$ is bounded from below and non-increasing, the limit

$$
V_{\infty}:=\lim _{t \rightarrow \infty} V\left(\boldsymbol{x}_{e}(t)\right)=\lim _{t \rightarrow \infty}\left(\frac{1}{2}\|\boldsymbol{v}\|_{\boldsymbol{M}}^{2}+\frac{1}{2}\|\boldsymbol{e}\|_{\boldsymbol{K}}^{2}\right)
$$


exists and lies in the interval $0 \leqslant V_{\infty} \leqslant V\left(\boldsymbol{x}_{\boldsymbol{e}}\left(t_{0}\right)\right)$. Note that $\|\boldsymbol{e}(t)\|_{\boldsymbol{K}}^{2}$ is absolutely continuous and $V\left(\boldsymbol{x}_{e}(t)\right)$ tends to an absolutely continuous (i.e., constant) function. Hence, $\|\boldsymbol{v}\|_{\boldsymbol{M}}^{2}$ must tend to an absolutely continuous function as well.

From (31) and (32) follows

$$
V_{\infty}-V\left(x_{e}^{-}\left(t_{0}\right)\right) \leqslant-\int_{t_{0}}^{t} W\left(x_{e}(\tau)\right) \mathrm{d} \tau .
$$

We deduce that $\lim _{t \rightarrow \infty} \int_{t_{0}}^{t} W\left(\boldsymbol{x}_{e}(\tau)\right) \mathrm{d} \tau=\lim _{t \rightarrow \infty} \int_{t_{0}}^{t}\|\boldsymbol{v}(\tau)\|_{\overline{\boldsymbol{C}}}^{2} \mathrm{~d} \tau<\infty$, because the left-hand side of (34) is finite. Because of the positive definiteness of $\overline{\boldsymbol{C}}$ and $\boldsymbol{M}$ together with the equivalence of norms, we obtain

$$
\lim _{t \rightarrow \infty} \int_{t_{0}}^{t}\|\boldsymbol{v}(\tau)\|_{M}^{2} \mathrm{~d} \tau<\infty
$$

The velocity error $\boldsymbol{v}(t)$ is discontinuous, and therefore we cannot invoke Barbalat's lemma [44]. This lemma is originally defined for uniformly continuous functions, but it can be extended to asymptotically absolutely continuous functions as presented in Proposition 3 in Appendix A. We can apply the extended lemma of Barbalat to (35) because $\|\boldsymbol{v}(t)\|_{\boldsymbol{M}}^{2}$ is asymptotically absolutely continuous and obtain

$$
\lim _{t \rightarrow \infty}\|\boldsymbol{v}(t)\|_{\boldsymbol{M}}^{2}=0
$$

The positive definiteness of $\boldsymbol{M}$ implies $\lim _{t \rightarrow \infty} \boldsymbol{v}(t)=\mathbf{0}$. Substituting the limit of $\boldsymbol{v}$ in (33) yields

$$
\lim _{t \rightarrow \infty} e(t)=c
$$

for some constant $\boldsymbol{c}$ satisfying $V\left(\boldsymbol{x}_{e}\left(t_{0}\right)\right) \geqslant \frac{1}{2}\|\boldsymbol{c}\|_{\boldsymbol{K}}^{2} \geqslant V_{\infty} \geqslant 0$.

Up to now, we have proven that the error dynamics, governed by the equality of measures

$$
\boldsymbol{M} \mathrm{d} \boldsymbol{v}+\boldsymbol{C} \boldsymbol{v} \mathrm{d} t+\boldsymbol{K} \boldsymbol{e} \mathrm{d} t=\boldsymbol{W}\left(\mathrm{d} \boldsymbol{P}_{1}-\mathrm{d} \boldsymbol{P}_{2}\right),
$$

is tending towards the limit point $\left(\boldsymbol{c}^{\mathrm{T}} \mathbf{0}\right)^{\mathrm{T}}$ for $t \rightarrow \infty$, where $\mathrm{d} \boldsymbol{P}_{1}=\boldsymbol{\lambda}_{1} \mathrm{~d} t+\boldsymbol{\Lambda}_{1} \mathrm{~d} \eta$ and $\mathrm{d} \boldsymbol{P}_{2}=$ $\lambda_{2} \mathrm{~d} t+\Lambda_{2} \mathrm{~d} \eta$ are the constraint impulse measures of the two considered solutions. Integrating the equality of measures (38) over the time interval $[t, t+\Delta t]$ yields

$$
\boldsymbol{M}\left(\boldsymbol{v}^{+}(t+\Delta t)-\boldsymbol{v}^{-}(t)\right)+\boldsymbol{C}(\boldsymbol{e}(t+\Delta t)-\boldsymbol{e}(t))+\boldsymbol{K} \int_{[t, t+\Delta t]} \boldsymbol{e}(\tau) \mathrm{d} \tau=\int_{[t, t+\Delta t]} \boldsymbol{W}\left(\mathrm{d} \boldsymbol{P}_{1}-\mathrm{d} \boldsymbol{P}_{2}\right),
$$

where $\Delta t>0$ is arbitrary. It proves useful to introduce the quantity $\tilde{\boldsymbol{\lambda}}_{\Delta t}(t)=\frac{1}{\Delta t} \int_{[t, t+\Delta t]}\left(\mathrm{d} \boldsymbol{P}_{1}-\right.$ $\mathrm{d} \boldsymbol{P}_{2}$ ), which can be regarded as the average constraint force of the error dynamics over the time lapse $[t, t+\Delta t]$. Subsequently, we take the limit $t \rightarrow \infty$ and use $\boldsymbol{v}(t) \rightarrow 0$ and $\boldsymbol{e}(t) \rightarrow \boldsymbol{c}$ for $t \rightarrow \infty$. The integrated equality of measures (divided by $\Delta t$ ) yields

$$
\boldsymbol{K} \boldsymbol{c}=\lim _{t \rightarrow \infty} \boldsymbol{W} \tilde{\boldsymbol{\lambda}}_{\Delta t}(t)=\lim _{t \rightarrow \infty} \sum_{i=1}^{m} \boldsymbol{w}_{i} \tilde{\lambda}_{\Delta t, i}(t),
$$

which is, in an averaged sense, the equilibrium of forces at infinity. The columns $\boldsymbol{w}_{i}$ of $\boldsymbol{W}$ are linearly independent from which we deduce that each of the $\operatorname{limits}_{\lim } \lim _{t \rightarrow \infty} \tilde{\lambda}_{\Delta t, i}(t)$ has to exist. The switching functions $\chi(t)$ are of class $\mathbb{K}^{m}$ by Assumption (A1), and it holds that $\lambda_{1, i}(t)=\lambda_{2, i}(t)=$ 0 and $\Lambda_{1, i}(t)=\Lambda_{2, i}(t)=0$ for $\chi_{i}(t)=0$. Therefore, for every time $t$ and every constraint $i$, there exists a $t_{i}^{*}>t$ for which $\lambda_{1, i}(t)=\lambda_{2, i}(t)=\Lambda_{1, i}(t)=\Lambda_{2, i}(t)=0 \forall t \in\left[t_{i}^{*}, t_{i}^{*}+\Delta t\right]$ if $\Delta t$ is taken small enough. If the constraint forces and impulses for both solutions vanish, then also the difference in the constraint impulse measures vanishes, and we obtain $\tilde{\lambda}_{\Delta t, i}\left(t_{i}^{*}\right)=0$. We conclude 
that each $\operatorname{limit}_{\lim _{t \rightarrow \infty}} \tilde{\lambda}_{\Delta t, i}(t)$ must vanish, and, according to (40) and $\boldsymbol{K}>0$, we obtain $\boldsymbol{c}=\mathbf{0}$. Therefore, $\boldsymbol{x}_{e}(t)=\mathbf{0}$ is globally uniformly attractively stable, which concludes the proof.

Uniform stability of all solution curves implies uniqueness of solutions and, which is an even stronger condition, continuous dependence on initial conditions. Generally, the solutions of a mechanical systems subjected to geometric unilateral constraints do not depend continuously on initial conditions as shown in [45]. However, this property does hold for the considered class of systems subjected to switched kinematic unilateral constraints as stated in the following corollary.

\section{Corollary 1}

The solution curves of system (24)-(28) for given switching functions $\chi(t)$ together with Assumptions (A1)-(A3) are unique and depend continuously on the initial conditions.

Proof

Consider two arbitrary solution curves $\boldsymbol{x}_{1}(t)=\varphi\left(t, t_{0}, \boldsymbol{x}_{10}\right)$ and $\boldsymbol{x}_{2}(t)=\varphi\left(t, t_{0}, \boldsymbol{x}_{20}\right)$ of the system. Uniform stability of all solutions curves of the system is proven in Theorem 1 . That is, for every $\varepsilon>0$, there exists a $\delta(\varepsilon)>0$, independent of $t_{0}$, such that

$$
\left\|x_{10}-x_{20}\right\|<\delta \quad \Longrightarrow \quad\left\|x_{1}(t)-x_{2}(t)\right\|<\varepsilon
$$

for all $t \geqslant t_{0}$. For fixed $t$ and $t_{0}$, we define the propagator by the mapping $\varphi_{t, t_{0}}\left(x_{0}\right):=\varphi\left(t, t_{0}, x_{0}\right)$. The uniform stability of all solutions implies that for every $\varepsilon>0$, there exists a $\delta(\varepsilon)>0$ such that

$$
\left\|x_{10}-x_{20}\right\|<\delta \quad \Longrightarrow\left\|\varphi_{t, t_{0}}\left(x_{10}\right)-\varphi_{t, t_{0}}\left(x_{20}\right)\right\|<\varepsilon,
$$

which is the Weierstrass continuity condition of the propagator. Uniqueness of solutions follows immediately from the continuous dependence on initial conditions.

We have seen in Section 3 that geometric unilateral constraints can be expressed using switched kinematic unilateral constraints if the switching functions $\chi(t)$ are generated by the constraint distances $\boldsymbol{g}$ rather than being an external input. We can state the following proposition concerning the boundedness of solutions of system (24)-(25) subjected to geometric unilateral constraints, that is, the force law and the impact law are given by (9) and (22).

\section{Proposition 2}

We consider system (24)-(25) with geometric unilateral constraints expressed by the force law (9), the impact law (22), and the local kinematic quantities given by (4). We assume that the impact map $\mathcal{H}_{g}$ is maximal monotone and fulfills the natural condition $\mathbf{0} \in \mathcal{H}_{g}(\mathbf{0})$. Furthermore, we assume that the external forcing fulfills Assumption (A2) and that the solutions depend continuously on initial conditions. Then, every solution of the considered system is bounded (in forward time), and there exists at least one solution that is bounded for all $t \in \mathbb{R}$.

Proof

For the boundedness condition, we aim to construct a globally attractive compact positively invariant set. Hereto, we consider the quadratic Lyapunov candidate function $V(\boldsymbol{q}, \boldsymbol{u})=$ $\frac{1}{2}\left\|\left(\begin{array}{l}\boldsymbol{q}-\boldsymbol{q}_{r} \\ \boldsymbol{u}\end{array}\right)\right\|_{\boldsymbol{P}}^{2}$ with $\boldsymbol{q}_{r}=-\boldsymbol{M}^{-1} \boldsymbol{W} \boldsymbol{G}^{-1} \boldsymbol{g}_{r}$ and $\boldsymbol{P}=\left(\begin{array}{cc}\boldsymbol{K} & \alpha \boldsymbol{M} \\ \alpha \boldsymbol{M} & \boldsymbol{M}\end{array}\right)$. The constant vector $\boldsymbol{q}_{r}$ fulfills $\boldsymbol{W}^{\mathrm{T}}\left(\boldsymbol{q}-\boldsymbol{q}_{r}\right)=\boldsymbol{g}$, which will be used in the following. Using (4) and (24), the density of the Lyapunov function w.r.t. $\mathrm{d} t$ is found as

$$
\dot{V}=-\left\|\left(\begin{array}{c}
\boldsymbol{q} \\
\boldsymbol{u}
\end{array}\right)\right\|_{\boldsymbol{Q}}^{2}+\alpha \boldsymbol{q}_{r}^{\mathrm{T}} \boldsymbol{K} \boldsymbol{q}-\boldsymbol{q}_{r}^{\mathrm{T}}(\boldsymbol{K}-\alpha \boldsymbol{C}) \boldsymbol{u}+(\alpha \boldsymbol{g}+\boldsymbol{\gamma})^{\mathrm{T}} \boldsymbol{\lambda}+\left(\alpha\left(\boldsymbol{q}-\boldsymbol{q}_{r}\right)+\boldsymbol{u}\right)^{\mathrm{T}} \boldsymbol{f},
$$

where $\boldsymbol{Q}=\left(\begin{array}{ll}\alpha \boldsymbol{K} & \frac{1}{2} \alpha \boldsymbol{C} \\ \frac{1}{2} \alpha \boldsymbol{C}^{\mathrm{T}} & \boldsymbol{C}-\alpha \boldsymbol{M}\end{array}\right)$. 
According to the Schur complement condition (Theorem 7.7.7 [46]), the matrix $\boldsymbol{P}$ is positive definite if $\alpha^{2}<\lambda_{\max }^{-1}(\boldsymbol{M}) \lambda_{\min }(\boldsymbol{K})=: c_{1}^{2}$, where $\lambda_{\max }(\boldsymbol{A})$ and $\lambda_{\min }(\boldsymbol{A})$ denote the maximal and minimal eigenvalues of a given matrix $\boldsymbol{A}$, respectively. The matrix $\boldsymbol{Q}$ is positive definite if $\alpha$ fulfills $\alpha>0$ and $\alpha<\lambda_{\min }(\overline{\boldsymbol{C}})\left(\lambda_{\max }(\boldsymbol{M})+\lambda_{\min }^{-1}(\boldsymbol{K}) \lambda_{\max }^{2}(\overline{\boldsymbol{C}})\right)^{-1}=: c_{2}$. Therefore, we choose $\alpha$ between $0<\alpha<\min \left(\left|c_{1}\right|, c_{2}\right)$ such that the matrices $\boldsymbol{P}$ and $\boldsymbol{Q}$ are positive definite.

The constraint forces $\lambda$ and the constraint velocities $\gamma$ as well as the constraint forces $\lambda$ and the constraint distances $\boldsymbol{g}$ are complementarity variables according to (9). Therewith, the density (43) can be bounded from above by

$$
\dot{V} \leqslant-\lambda_{\min }(\boldsymbol{Q})\left(\|\boldsymbol{q}\|^{2}+\|\boldsymbol{u}\|^{2}\right)+\beta_{1}\|\boldsymbol{q}\|+\beta_{2}\|\boldsymbol{u}\|+\beta_{3},
$$

where $\beta_{1}=\alpha\left(\left\|\boldsymbol{K} \boldsymbol{q}_{r}\right\|+f_{\max }\right), \beta_{2}=\left\|\left(\boldsymbol{K}-\alpha \boldsymbol{C}^{\mathrm{T}}\right) \boldsymbol{q}_{r}\right\|+f_{\max }$, and $\beta_{3}=\alpha\left\|\boldsymbol{q}_{r}\right\| f_{\max }$. The quadratic term in (44) will dominate far away from the origin, which implies the existence of a bound $\delta_{1}>0$ such that $\dot{V}<0$ for all $\|\boldsymbol{q}\|+\|\boldsymbol{u}\|>\delta_{1}$.

The change of the Lyapunov function due to impacts is given by

$$
V^{+}-V^{-}=\left(\begin{array}{l}
\left(\boldsymbol{q}-\boldsymbol{q}_{r}\right) \\
\frac{1}{2}\left(\boldsymbol{u}^{+}+\boldsymbol{u}^{-}\right)
\end{array}\right)^{\mathrm{T}}\left(\begin{array}{c}
\alpha \boldsymbol{M}\left(\boldsymbol{u}^{+}-\boldsymbol{u}^{-}\right) \\
\boldsymbol{M}\left(\boldsymbol{u}^{+}-\boldsymbol{u}^{-}\right)
\end{array}\right)=\alpha \boldsymbol{g}^{\mathrm{T}} \boldsymbol{\Lambda}+\bar{\gamma}^{\mathrm{T}} \boldsymbol{\Lambda},
$$

where (4) and (25) have been used. The constraint impulse $\Lambda_{i}$ of a constraint $i$ vanishes for $g_{i}>0$. The impulse $\Lambda_{i}$ may only be positive if the constraint distance $g_{i}=0$ vanishes and, thus, $\boldsymbol{\Lambda}$ and $\boldsymbol{g}$ are complementarity variables. Furthermore, the impacts are dissipative, according the maximal monotonicity of $\mathcal{H}_{g}$ together with the natural condition, which yields $V^{+}-V^{-} \leqslant 0$.

We have found that the differential measure of $V$ fulfills $\mathrm{d} V<0$ for all $\|\boldsymbol{q}\|+\|\boldsymbol{u}\|>\delta_{1}$.

Let $\delta_{2}=\max _{\|\boldsymbol{q}\|+\|\boldsymbol{u}\| \leqslant \delta_{1}} V(\boldsymbol{q}, \boldsymbol{u})$. Consequently, the set $\left\{\left(\begin{array}{c}\boldsymbol{q} \\ \boldsymbol{u}\end{array}\right) \in \mathbb{R}^{2 f} \mid V(\boldsymbol{q}, \boldsymbol{u}) \leqslant \delta_{2}\right\}$ is compact positively invariant, and every solution starting outside of this set will approach it in forward time. Therefore, every solution is bounded in forward time. Furthermore, the existence of a compact positive invariant set together with continuous dependence on initial conditions of the solutions curves implies the existence of a bounded steady-state solution. This conclusion follows from Proposition 4 presented in Appendix B, where the corresponding lemma of Yakubovich is extended to measure differential inclusions.

For Proposition 2, the condition of maximal monotonicity together with the natural condition can be relaxed to dissipativity of the impact map. The condition of continuous dependence on initial conditions is generally difficult to be checked [17]. The system considered in Proposition 2 without external forcing admits a stable equilibrium as shown in [47]. Note that Proposition 2 is generally not valid for systems subjected to switched kinematic unilateral constraints even if the impact map is maximal monotone. Generally, there exist unbounded solutions of system (24)-(28) if the switching functions $\chi(t)$ do not fulfill Assumption (A1).

The existence of a bounded solution of an a.i.s. system imply the boundedness of all solutions. The boundedness of solutions is not only interesting in itself but is also necessary to define the error using the difference of solutions. However, Proposition 2 does not imply the existence of a bounded solution for system (24)-(28), and therefore the convergence property of the system subjected to switched kinematic unilateral constraints cannot be deduced.

\section{OBSERVER DESIGN}

The property of attractive incremental stability is used in this section to design a state observer using master-slave synchronization. The master system is subjected to geometric unilateral constraints, whereas the slave system is a perfect replica of the master system with one-way clutch constraints that are switched on when the corresponding constraints of the master system are closed. If the slave system is initialized with the same initial conditions as the master system, then the motion of the slave system is an exact copy of the master system. Under the conditions of Theorem 1, the slave system is a.i.s. because of the switched kinematic unilateral constraints. The attractive incremental 


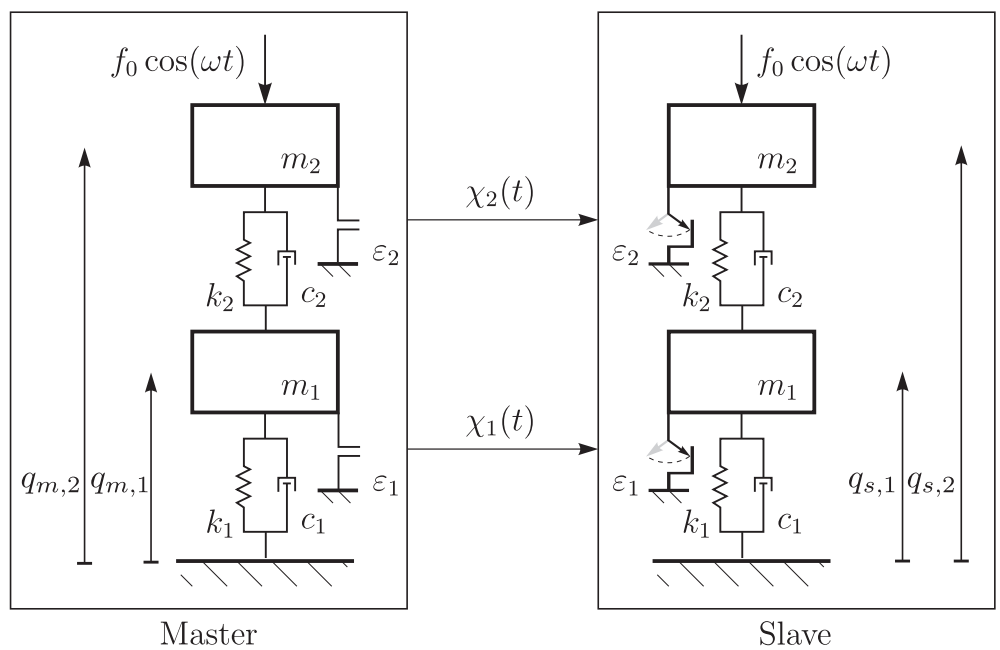

Figure 2. Master-slave system coupled by the Boolean switching functions $\chi(t)$ for a forced double mass impact oscillator. The master system (left) is subjected to geometric unilateral constraints, and the slave system (right) is subjected to switched kinematic unilateral constraints.

stability implies that all solutions of the slave system converge to each other and also to the motion in synchrony with the master system. Therefore, the synchronization error tends to zero, and the full state of the master system is reconstructed by the slave system.

\section{Example 1 (Double mass impact oscillator)}

We illustrate the synchronization-based observer using the example of a double mass impact oscillator as depicted in Figure 2. The oscillator consists of two masses coupled by spring-damper elements, and the upper mass is externally forced. The master system and the slave system are modeled as forced linear time-invariant Lagrangian systems subjected to geometric and switched kinematic unilateral constraints, respectively. However, the geometric unilateral constraints of the master system can be envisaged as switched kinematic unilateral constraints for which the switching functions are internally generated by the constraint distances. The constraints of the slave system are switched on whenever the corresponding constraint distances of the master system vanish.

The dynamics of the master system as well as the slave system are described by (24)-(27) with

$$
\begin{aligned}
& \boldsymbol{M}=\left(\begin{array}{ll}
m_{1} & 0 \\
0 & m_{2}
\end{array}\right), \boldsymbol{C}=\left(\begin{array}{ll}
c_{1}+c_{2} & -c_{2} \\
-c_{2} & c_{2}
\end{array}\right), \boldsymbol{K}=\left(\begin{array}{ll}
k_{1}+k_{2} & -k_{2} \\
-k_{2} & k_{2}
\end{array}\right), \boldsymbol{W}=\left(\begin{array}{ll}
1 & 0 \\
0 & 1
\end{array}\right), \\
& \boldsymbol{f}(t)=\left(\begin{array}{l}
0 \\
1
\end{array}\right) f_{0} \cos (\omega t) .
\end{aligned}
$$

The generalized force directions are linearly independent, and the system matrices $\boldsymbol{M}, \boldsymbol{C}$ and $\boldsymbol{K}$ are symmetric and positive definite. The impacts are modeled using the generalized Poisson's impact law. The coefficients of restitution are chosen equal to ensure the property of maximal monotonicity of the impact law. The harmonic excitation $\boldsymbol{f}(t)$ is bounded by $\sup _{t \in \mathbb{R}}\|\boldsymbol{f}(t)\|=f_{0}<\infty$. The system parameters of the master system are chosen such that its solution moves on a chaotic attractor for which both contacts keep on opening and closing in a chaotic way.

The constraints may repeatedly experience accumulation points and stay closed during some time intervals, but they will always open again eventually. Furthermore, for both constraints, there exists a $\Delta t>0$ such that for every $t$, there exists a $t^{*}(t)>t$ such that the constraint is open during the interval $\left[t^{*}, t^{*}+\Delta t\right]$ because of the chaotic motion. Therefore, the switching functions $\chi(t)$, which are fed to the slave system, are of class $\mathbb{K}^{2}$. The necessary Assumptions (A1)-(A3) in Section 6 are fulfilled, and attractive incremental stability of the slave system follows from Theorem 1.

Let us consider the Lyapunov function 

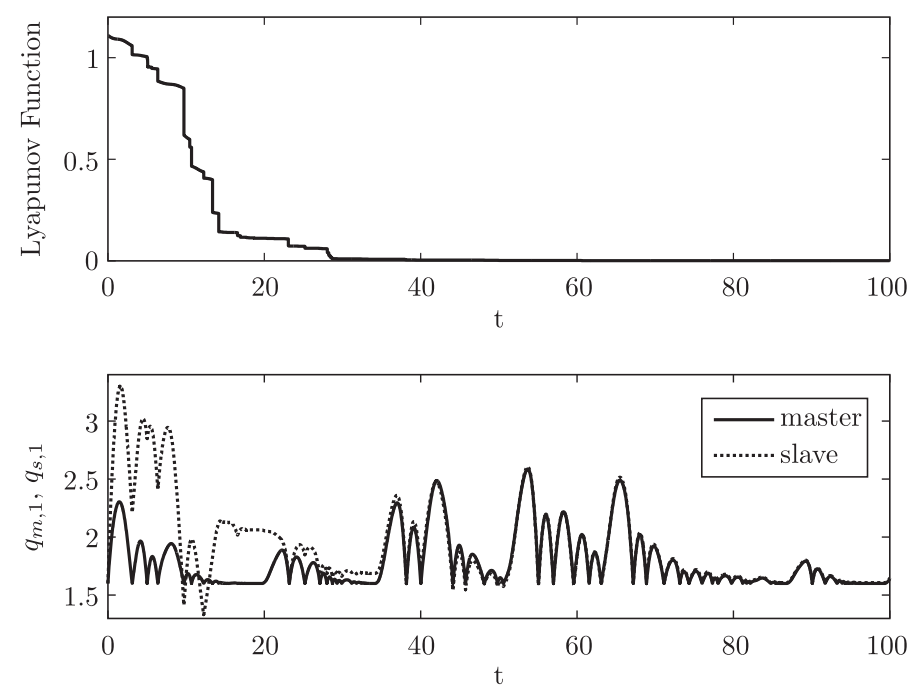

Figure 3. Lyapunov function (top) and the first generalized coordinate of the master system and the slave system (bottom) for the coupled double mass impact oscillator.

$$
V(t)=\frac{1}{2}\left\|\boldsymbol{u}_{m}-\boldsymbol{u}_{s}\right\|_{\boldsymbol{M}}^{2}+\frac{1}{2}\left\|\boldsymbol{q}_{m}-\boldsymbol{q}_{s}\right\|_{\boldsymbol{K}}^{2},
$$

which captures the mechanical energy of the error between the master system (index $m$ ) and the slave system (index $s$ ). It has been shown that this Lyapunov function tends to zero as $t$ tends to infinity for every choice of initial conditions, and we have $\lim _{t \rightarrow \infty} \boldsymbol{q}_{s}(t)-\boldsymbol{q}_{m}(t)=\mathbf{0}$ and $\lim _{t \rightarrow \infty} \boldsymbol{u}_{s}(t)-\boldsymbol{u}_{m}(t)=\mathbf{0}$. Synchronization is achieved using only the Boolean contact information of the master system without any continuous measurements. The state of the slave system can directly be used as state estimate of the master system.

The Lyapunov function $V(t)$ and the first generalized coordinate of each subsystem, that is, $q_{m, 1}$ and $q_{s, 1}$, are depicted in Figure 3 for a simulation with the set of parameters $m_{1}=m_{2}=2 \mathrm{~kg}$, $k_{1}=k_{2}=1 \mathrm{~N} / \mathrm{m}, c_{1}=c_{2}=0.02 \mathrm{Ns} / \mathrm{m}, g_{r}=-(1.61 .5)^{\mathrm{T}} \mathrm{m}, f_{0}=5 \mathrm{~N}, \omega=3 \mathrm{rad} / \mathrm{s}$, and $\varepsilon_{1}=\varepsilon_{2}=0.8$ and the initial conditions $\boldsymbol{q}_{m 0}=\left(\begin{array}{lll}1.6 & 1.8\end{array}\right)^{\mathrm{T}} \mathrm{m}, \boldsymbol{u}_{m 0}=\left(\begin{array}{ll}1 & 1\end{array}\right)^{\mathrm{T}} \mathrm{m} / \mathrm{s}, \boldsymbol{q}_{s 0}=$ $\left(\begin{array}{lll}1.6 & 1.6\end{array}\right)^{\mathrm{T}} \mathrm{m}$, and $\boldsymbol{u}_{s 0}=\left(\begin{array}{ll}2 & 1.3\end{array}\right)^{\mathrm{T}} \mathrm{m} / \mathrm{s}$.

The solution of the master system is chaotic such that solutions starting nearby diverge rapidly. Note that the master system does not enjoy the property of incremental stability because it is subjected to geometric unilateral constraints. Figure 3 shows that discontinuities are present in the evolution of the Lyapunov function at impact time instants. Impacts are not essential for synchronization if $C \succ 0$ but improve the synchronization rate. The Lyapunov function is non-increasing, but it is not strictly decreasing as the mechanical system is not strictly passive. Nevertheless, the Lyapunov function asymptotically tends to zero as $t$ goes to infinity and synchronization between the master system and the slave system is achieved also in the presence of accumulation points.

\section{Example 2 (Impacting beam)}

As a second example, we consider a forced beam with a unilateral support as depicted in Figure 4. The vertical displacement $w(t, x)$ is measured with respect to a solid frame, which itself is excited by $e(t)=e_{0} \cos \omega t$. The support is at the location $x_{c}$, which corresponds to a node of the third eigenmode, and the contact is closed for $w\left(t, x_{c}\right)=0$. The beam is modeled as a plane linearized Euler-Bernoulli beam for which the rotational inertia is neglected. In this case, the virtual work is given by

$$
0=\delta W=\int_{0}^{l}\left(\delta w^{\prime \prime} E I w^{\prime \prime}+\delta w A \rho \ddot{w}+\delta w d(\dot{w}-\dot{e})\right) \mathrm{d} x-\delta w\left(x_{c}\right) \lambda \quad \forall \delta w \mid \delta w(0)=0
$$




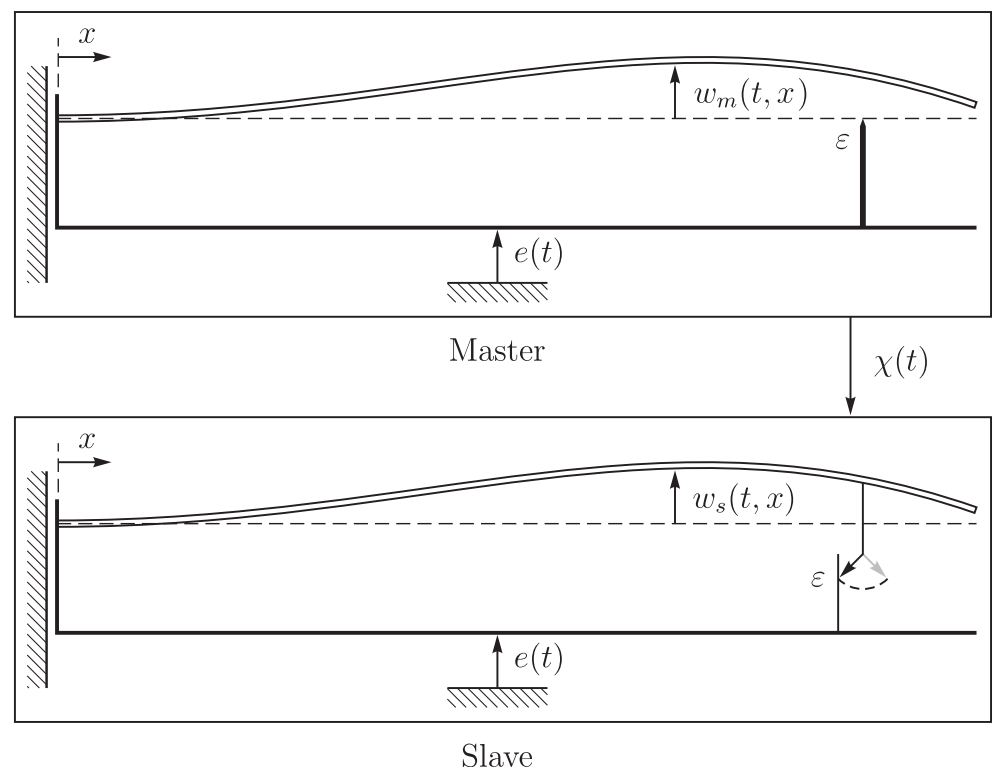

Figure 4. Master-slave system coupled by the Boolean switching function $\chi(t)$ for the impacting beam model. The master system (top) is subjected to a geometric unilateral constraint, and the slave system (bottom) is subjected to a switched kinematic unilateral constraint.

with length of the beam $l$, elastic modulus $E$, second moment of area $I$, density $\rho$, cross-sectional area $A$, and damping coefficient $d$. The damping is modeled as linear viscous damping with the frame as reference. Even though there are physically more intuitive ways to model the damping effects, this choice is made because it leads to the same decay rate for all eigenmodes.

The first three eigenmodes of the free beam are given by

$$
v_{i}(x)=\cos \beta_{i} x-\cosh \beta_{i} x+\frac{\cos \beta_{i} l+\cosh \beta_{i} l}{\sin \beta_{i} l+\sinh \beta_{i} l}\left(-\sin \beta_{i} x+\sinh \beta_{i} x\right) \quad \text { for } i \in\{1,2,3\},
$$

where $\beta_{i}$ is the $i$-th root of $\cos \beta_{i} l \cosh \beta_{i} l+1=0$. We proceed with the Galerkin approach by approximating the displacement $w(t, x)$ and the virtual displacements $\delta w(t, x)$ using the first three eigenmodes as

$$
\begin{aligned}
& w(t, x) \approx w_{n}(t, x)=\boldsymbol{v}(x)^{\mathrm{T}} \boldsymbol{q}(t)+e(t)+\text { const. } \\
& \delta w(t, x) \approx \delta w_{n}(t, x)=\boldsymbol{v}(x)^{\mathrm{T}} \delta \boldsymbol{q}(t),
\end{aligned}
$$

where $\boldsymbol{q}(t)$ are the generalized coordinates and $\delta \boldsymbol{q}(t)$ are the corresponding virtual displacements. Substituting the approximation (50) into (48) yields

$$
0=\delta \boldsymbol{q}^{\mathrm{T}}(\underbrace{\int_{0}^{l} \boldsymbol{v}^{\prime \prime} E I \boldsymbol{v}^{\prime \prime \mathrm{T}} \mathrm{d} x}_{\boldsymbol{K}}+\underbrace{\int_{0}^{l} \boldsymbol{v} A \rho \boldsymbol{v}^{\mathrm{T}} \mathrm{d} x}_{\boldsymbol{M}} \ddot{\ddot{q}}+\underbrace{\int_{0}^{l} \boldsymbol{v} A \rho \mathrm{d} x \ddot{e}}_{-\boldsymbol{f}(t)}+\underbrace{\int_{0}^{l} \boldsymbol{v} d \boldsymbol{v}^{\mathrm{T}} \mathrm{d} x}_{\boldsymbol{C}} \dot{\boldsymbol{q}}-\underbrace{\boldsymbol{v}\left(x_{c}\right)}_{\boldsymbol{W}} \lambda) \forall \delta \boldsymbol{q} .
$$

The impacting beam model can therefore be written in the form (24)-(27) with

$$
\begin{aligned}
& \boldsymbol{M}=\rho A l\left(\begin{array}{lll}
1 & 0 & 0 \\
0 & 1 & 0 \\
0 & 0 & 1
\end{array}\right), \boldsymbol{C}=d l\left(\begin{array}{lll}
1 & 0 & 0 \\
0 & 1 & 0 \\
0 & 0 & 1
\end{array}\right), \boldsymbol{K}=\frac{E I}{l^{3}}\left(\begin{array}{ccc}
\left(\beta_{1} l\right)^{4} & 0 & 0 \\
0 & \left(\beta_{2} l\right)^{4} & 0 \\
0 & 0 & \left(\beta_{3} l\right)^{4}
\end{array}\right), \boldsymbol{W}=\boldsymbol{v}\left(x_{c}\right), \\
& \text { and } \boldsymbol{f}(t)=\left(\begin{array}{l}
f_{1}(t) \\
f_{2}(t) \\
f_{3}(t)
\end{array}\right), \text { where } f_{i}(t)=-2 \frac{\rho A l}{\beta_{i} l} \frac{\cos \beta_{i} l+\cosh \beta_{i} l}{\sin \beta_{i} l+\sinh \beta_{i} l} e_{0} \omega^{2} \cos \omega t \quad \text { for } i \in\{1,2,3\} .
\end{aligned}
$$



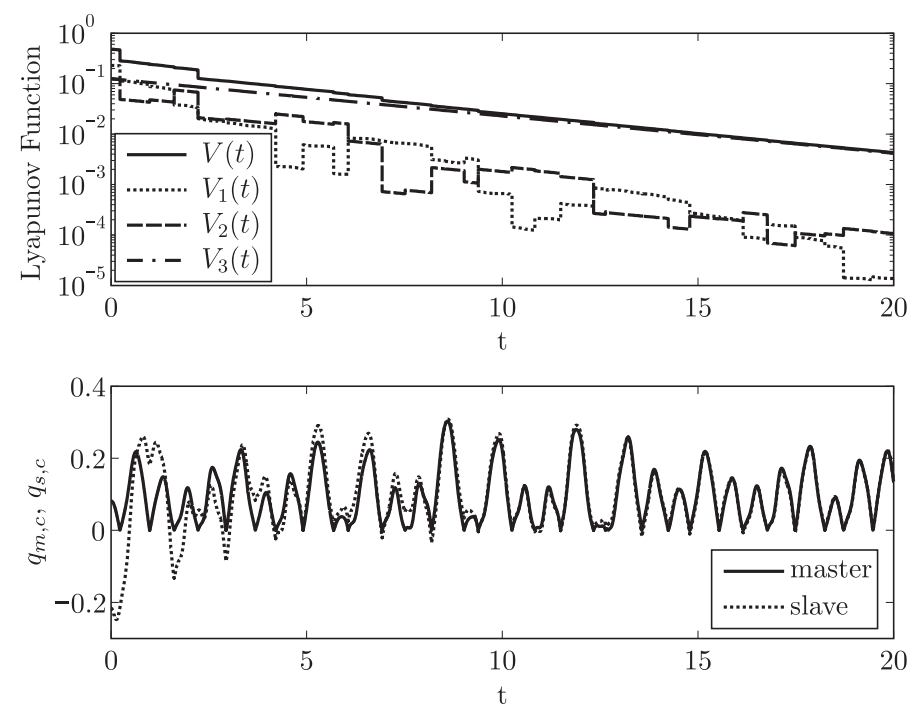

Figure 5. Lyapunov function $V(t)$ and the energy contents $V_{i}(t)$ of the first three eigenmodes (top) and the time evolution of the vertical displacement at the location of the support (bottom) for the impacting beam model.

We consider the same master-slave synchronization setup for the state observer design as proposed at the beginning of this section. The impacts are modeled using the generalized Poisson's impact law. Assumptions (A1) and (A3) are fulfilled using the same reasoning as in Example 1, and the excitation $\boldsymbol{f}(t)$ meets Assumption (A2) because max $|\ddot{e}(t)|=\omega^{2} e_{0}<\infty$. Therefore, the slave system is a.i.s, and the Lyapunov function (47) capturing the mechanical energy of the error dynamics tends to zero. The Lyapunov function can be split up into the energy contents of each eigenmode as

$$
V(t)=\sum_{i=1}^{3} V_{i}(t), \quad \text { where } V_{i}(t)=\frac{1}{2} \rho A l\left(u_{m i}-u_{s i}\right)^{2}+\frac{1}{2} \frac{E I}{l^{3}}\left(\beta_{i} l\right)^{4}\left(q_{m i}-q_{s i}\right)^{2} .
$$

The time evolution of the Lyapunov function $V(t)$ and the energy content $V_{1}(t), V_{2}(t), V_{3}(t)$ of the eigenmodes are depicted in Figure 5. The Lyapunov function is plotted using a logarithmic axis for a simulation with the set of parameters $\varepsilon=0.7, \rho A l=1 \mathrm{~kg}, \frac{E I}{l^{3}}=1 \mathrm{~N} / \mathrm{m}, d l=0.085 \mathrm{Ns} / \mathrm{m}, e_{0}=$ $0.03 \mathrm{~m}, \omega=9.5 \mathrm{rad} / \mathrm{s}, g_{r}=0 \mathrm{~m}, \boldsymbol{q}_{m 0}=\left(\begin{array}{lll}0.05 & 0 & 0\end{array}\right)^{\mathrm{T}} \mathrm{m}, \boldsymbol{q}_{s 0}=-\left(\begin{array}{lll}0.14 & 0.023 & 0.0081\end{array}\right)^{\mathrm{T}} \mathrm{m}$, and $\boldsymbol{u}_{m 0}=\boldsymbol{u}_{s 0}=\left(\begin{array}{lll}0 & 0 & 0\end{array}\right)^{\mathrm{T}} \mathrm{m} / \mathrm{s}$.

Figure 5 also shows the time evolution of the vertical displacement $w_{m}\left(t, x_{c}\right)$ and $w_{s}\left(t, x_{c}\right)$ for the master system and the slave system at the location of the support. The vertical displacement of the master system is restricted to $w_{m}\left(t, x_{c}\right) \geqslant 0$ because of the geometric unilateral constraint. The slave system, however, is subjected to a switched kinematic unilateral constraint, and therefore, there is no restriction on the sign of the vertical displacement $w_{s}\left(t, x_{c}\right)$.

During non-impulsive motion, the eigenmodes are decoupled, and their energy is non-increasing because of the positive definiteness of the damping matrix $\boldsymbol{C}$. The only coupling of the eigenmodes is due to the support. At collision time instants, energy may transfer between the first two eigenmodes. The energy content of the first and second eigenmodes, that is, $V_{1}(t)$ and $V_{2}(t)$, may increase at impacts, but the sum is strictly decreasing. The third eigenmode is not influenced by the impact because the support is chosen to be located at a node of this eigenmode, and it is therefore decoupled from the other eigenmodes.

The attractive incremental stability property of the impacting beam model is due to the maximal monotonicity of the impact law and the internal damping. This property persists for an arbitrary number of eigenmodes (or generally Ansatz functions) and other models for the internal damping as long as the system matrices remain positive definite. 


\section{CONCLUSIONS}

An observer design for Lagrangian systems subjected to (multiple) unilateral constraints has been presented, which uses only the information of the impact time instants. Of prime importance are the new concept of switched kinematic unilateral constraints and the maximal monotonicity property of the impact map. The switched kinematic unilateral constraints ensure that the discontinuities of the observer system and the observed system occur at the same time instants. The maximal monotonicity of the impact map guarantees that the distance between the considered solutions is non-increasing at impact time instants.

The observer proposed in the paper is applicable to passive systems which are not necessarily strictly passive. Other approaches assume strict passivity which is enforced through continuous measurements of the state, for example, as in the case of a Luenberger observer. The novelty of our approach is that mechanical systems are considered, which are typically only passive, and no continuous measurements of the state are used. Furthermore, the presented state observer is, strictly speaking, a state detector as the observed system may have non-observable states that are unaffected by any unilateral constraints.

The master-slave synchronization approach, which has been introduced in this paper, where the Boolean information captures which contacts of the master system are open or closed, is fed into the slave system. This approach is no longer applicable if the coupling is inherently bidirectional, for example, in the case of two identical impact oscillators coupled by a spring-damper element.

The solution of the slave system (observer) tends to the solution of the observed system for every initial condition also in the presence of accumulation points. Synchronization is guaranteed because the slave system is globally a.i.s. and the solutions of both systems are identical if the slave system is started using the initial conditions of the master system. If the slave system is a.i.s. and admits a steady-state solution, then it is uniformly convergent. The property of uniform convergence has generally useful implications such as uniqueness of the steady-state solution or that a periodic input implies a periodic output. In observer design, however, the focus lies on tracking the reference trajectory generated by the observed system, and uniform convergence is therefore not a necessary requirement.

In the foregoing, the constraint distances are assumed to be affine and autonomous, but the developed results on attractive incremental stability can directly be generalized to the non-autonomous case. Furthermore, only the time information of the impacts of the master system is used so far, while the value of the (vanishing) constraint distances is unused. This additional information could be used to achieve faster (or finite time [48]) synchronization. The idea is to allow for position jumps of the slave system, which is feasible, because the slave system is not a physical system. The generalized coordinates of the slave system are in that case no longer absolutely continuous but rather special functions of locally bounded variation. The extension to position jumps is part of further research.

\section{ACKNOWLEDGEMENTS}

The idea to extend the proof of Yakubovich to measure differential inclusions with continuous dependence on initial conditions stems from Nathan van de Wouw (Eindhoven University of Technology). This research is supported by Swiss National Science Foundation through the project 'Synchronization of Dynamical Systems with Impulsive Motion' (SNF 200021-144307).

\section{APPENDIX A: EXTENSION OF BARBALAT'S LEMMA}

The lemma of Barbalat is originally defined for uniformly continuous functions, and it is often used for invariance-like theorems. Here, we extend Barbalat's lemma for the special functions of locally bounded variation, which are asymptotically absolutely continuous. These functions approach an absolutely continuous function asymptotically, and therefore the atomic measure tends to zero. 
Definition 5

A function $f: \mathbb{R} \mapsto \mathbb{R}$ of special locally bounded variation is called asymptotically absolutely continuous if there exists an absolutely continuous function $g: \mathbb{R} \mapsto \mathbb{R}$ such that

$$
\lim _{t \rightarrow \infty}|f(t)-g(t)|=0 .
$$

The following extension of Barbalat's lemma is based on the proof presented in [44].

Proposition 3 (Barbalat's lemma for asymptotically absolutely continuous functions)

Let $f: \mathbb{R} \mapsto \mathbb{R}$ be a special function of locally bounded variation on $[0, \infty)$, which is asymptotically absolutely continuous. If the limit

$$
\lim _{T \rightarrow \infty} \int_{0}^{T} f(t) \mathrm{d} t
$$

exists and is finite, then

$$
\lim _{t \rightarrow \infty} f(t)=0 .
$$

Proof

We will establish a proof by contradiction. Let us assume that (A.3) does not hold; then $\exists c_{1}>$ $0 \forall T_{1}>0 \exists t>T_{1}:|f(t)| \geqslant c_{1}$. Because the function $f$ is asymptotically absolutely continuous, there exists an absolutely continuous function $g$ such that $\exists T_{2}>0:|f(t)-g(t)|<\frac{c_{1}}{4}$ for almost all $t>T_{2}$. Therefore, there exists a time $\hat{t}>\max \left\{T_{1}, T_{2}\right\}$ with $|f(\hat{t})| \geqslant c_{1}$ and $|f(t)-g(t)|<\frac{c_{1}}{4}$ for almost all $t>\hat{t}$. Because of the absolute continuity of $g(t)$, there exists a time interval $\Delta t>0$ such that $|g(t)-g(\hat{t})|<\frac{c_{1}}{4} \forall t \in[\hat{t}, \hat{t}+\Delta t]$. Together with the triangle inequality and the fact that $f(t)$ has the same sign for almost all $t \in[\hat{t}, \hat{t}+\Delta t]$, we obtain

$$
\begin{aligned}
\left|\int_{\hat{t}}^{\hat{t}+\Delta t} f(t) \mathrm{d} t\right| & =\int_{\hat{t}}^{\hat{t}+\Delta t}|f(t)| \mathrm{d} t \\
& \geqslant \int_{\hat{t}}^{\hat{t}+\Delta t}|f(\hat{t})|-|f(\hat{t})-g(\hat{t})|-|g(\hat{t})-g(t)|-|g(t)-f(t)| \mathrm{d} t \\
& >\int_{\hat{t}}^{\hat{t}+\Delta t} c_{1}-\frac{c_{1}}{4}-\frac{c_{1}}{4}-\frac{c_{1}}{4} \mathrm{~d} t \\
& =\frac{c_{1}}{4} \Delta t .
\end{aligned}
$$

For every $T_{1}>0$, there exists a $\hat{t}>T_{1}$ for which (A.4) holds. This is in contradiction with the existence of the finite limit (A.2).

\section{APPENDIX B: EXISTENCE OF A STEADY-STATE SOLUTION}

In [10], Yakubovich proves a lemma that formulates that if a non-autonomous differential inclusion (or discontinuous differential equation with solutions in the sense of Filippov) with bounded right-hand sides exhibits a compact positively invariant $\operatorname{set} \mathcal{C}$, then the existence of a solution that is bounded for all $t \in \mathbb{R}$ is guaranteed. The differential inclusions considered in [10] are not guaranteed to have uniqueness of solutions in forward (and backward) time. Consequently, the solutions of the considered differential inclusions do not enjoy continuity with respect to the initial condition. The technical difficulties that arise because of the non-uniqueness of solutions are handled in the proof of [10] by using an elegant choice of subsequences of solution curves. The non-uniqueness of solutions results in a set-valued propagator $\boldsymbol{\varphi}_{t, t_{0}}\left(\boldsymbol{x}_{0}\right)$, which maps each point $\boldsymbol{x}_{0}$ in the state space at time $t_{0}$ to a set of points at time $t$. Although not explicitly mentioned in [10], an essential ingredient in the proof of [10] is that the propagator of the considered differential inclusion is an upper 
semicontinuous set-valued map with compact images (see also [49], Theorem 4.11). Hence, every compact subset $\mathcal{K}$ of the state space is mapped by the propagator to a compact subset $\varphi_{t, t_{0}}(\mathcal{K})$ of the state space. Furthermore, the proof in [10] also relies on the fact that the solutions of differential inclusions are absolutely continuous in time.

In [16], it has been suggested that the lemma of Yakubovich may be extended to measure differential inclusions, for which the solution curves are discontinuous in time. Here, we make this assertion more precise. In the following, the lemma of [10] will be modified such that it is valid for a certain class of dynamical systems, which are characterized by the following properties:

(P1) For each point $\boldsymbol{x}_{0}$ in the admissible subset $\mathcal{A}$ of the state space $\mathbb{R}^{2 f}$ and for each time instant $t_{0} \in \mathbb{R}$, there exists a unique forward solution curve $\varphi\left(t, t_{0}, x_{0}\right) \in \mathcal{A}$ for almost all $t \geqslant t_{0}$.

(P2) The solution curve $\varphi\left(t, t_{0}, x_{0}\right)$ may be discontinuous in time $t$ but is of locally bounded variation.

(P3) The propagator, defined by the mapping $\varphi_{t, t_{0}}\left(x_{0}\right)=\varphi\left(t, t_{0}, x_{0}\right)$, is a (single valued) continuous map. That is, the solutions are continuous with respect to the initial condition $\boldsymbol{x}_{0}$.

The Property (P2) implies that the left limit $\varphi^{-}\left(t, t_{0}, x_{0}\right)$ and the right limit $\varphi^{+}\left(t, t_{0}, x_{0}\right)$ exist for all $t \in \mathbb{R}$. The following modified lemma is on the one hand more general as it also applies to measure differential inclusions and on the other hand more restrictive because it is only applicable to systems with continuity with respect to the initial condition (and therefore uniqueness in forward time).

Proposition 4 (Modification of Yakubovich [10], Lemma 2)

Consider a dynamical system enjoying the properties (P1)-(P3). If $\mathcal{C} \subset \mathbb{R}^{n}$ is a compact positively invariant set such that

$$
x_{0} \in \mathcal{C} \Rightarrow \varphi\left(t, t_{0}, x_{0}\right) \in \mathcal{C} \quad \text { for almost all } t \geqslant t_{0}
$$

independent of $t_{0}$, then there exists a solution curve $\overline{\boldsymbol{x}}(t) \in \mathcal{C}$ for almost all $t \in \mathbb{R}$.

Proof

We set $\mathcal{C}_{0}=\mathcal{C}$ and denote by $\mathcal{C}_{1}$ the set of all points $\boldsymbol{x} \in \mathbb{R}^{n}$, which are the images of the propagator $\varphi_{0,-1}^{+}\left(x_{0}\right)=\varphi^{+}\left(0,-1, x_{0}\right)$ with $x_{0} \in \mathcal{C}_{0}$ :

$$
\mathcal{C}_{1}:=\left\{x \in \mathbb{R}^{n} \mid x=\varphi^{+}\left(0,-1, x_{0}\right), x_{0} \in \mathcal{C}_{0}\right\},
$$

or we may use the short-hand notation $\mathcal{C}_{1}=\varphi^{+}\left(0,-1, \mathcal{C}_{0}\right)$. It holds that $\mathcal{C}_{1} \subset \mathcal{C}_{0}$ because $\mathcal{C}_{0}$ is positively invariant. Similarly, we define the sets $\mathcal{C}_{j}=\varphi^{+}\left(0,-j, \mathcal{C}_{0}\right)$. The sets $\mathcal{C}_{j}$ are generally not positively invariant, but they are nested. Namely, if $A \subset B$, then it holds for any continuous mapping $f$ that $f(A) \subset f(B)$. Similarly, because $\varphi^{+}\left(-j,-(j+1), \mathcal{C}_{0}\right) \subset \mathcal{C}_{0}$ because of the positive invariance of $\mathcal{C}_{0}$ and because of the definition of the sets $\mathcal{C}_{j}$, it holds that $\mathcal{C}_{j+1} \subset \mathcal{C}_{j}$. That is, the sets are nested such that

$$
\mathcal{C}_{0} \supset \mathcal{C}_{1} \supset \mathcal{C}_{2} \supset \ldots
$$

The existence of solution curves implies that the sets $\mathcal{C}_{j}$ are nonempty. Furthermore, for any continuous mapping $f$, it holds that if $A$ is compact, then also $f(A)$ is compact. The propagator $\varphi_{t, t_{0}}\left(x_{0}\right)$ is assumed to be continuous with respect to the initial point $x_{0}$, and therefore the sets $\mathcal{C}_{j}=\varphi^{+}\left(0,-j, \mathcal{C}_{0}\right)$ are compact. From (B.3) and the fact that the sets $\mathcal{C}_{j}$ are nonempty and compact, it follows that there is a point $\boldsymbol{a}_{0} \in \bigcap \mathcal{C}_{j}$. The solution curve $\varphi\left(t, 0, \boldsymbol{a}_{0}\right)$ remains in the positively invariant set $\mathcal{C}_{0}$ for almost all $t \geqslant 0$ because $\boldsymbol{a}_{0} \in \mathcal{C}_{0}$. Moreover, $\boldsymbol{a}_{0} \in \mathcal{C}_{j}$ implies that there exists a point $\boldsymbol{a}_{j} \in \mathcal{C}_{0}$ and a solution curve $\overline{\boldsymbol{x}}(t)=\boldsymbol{\varphi}\left(t,-j, \boldsymbol{a}_{j}\right)$ with $\overline{\boldsymbol{x}}(0)=\boldsymbol{a}_{0}$ such that $\overline{\boldsymbol{x}}(t) \in \mathcal{C}_{0}$ for almost all $t \geqslant-j$. Because $j$ is an arbitrary integer, the solution curve fulfills $\overline{\boldsymbol{x}}(t) \in \mathcal{C}$ for almost all $t \in \mathbb{R}$. 


\section{REFERENCES}

1. Yoshizawa T. Stability Theory and the Existence of Periodic Solutions and Almost Periodic Solutions. Springer-Verlag: New York, 1975.

2. Fromion V, Monaco S, Normand-Cyrot D. Asymptotic properties of incrementally stable systems. IEEE Transactions on Automatic Control 1996; 41:721-723.

3. Fromion V, Scorletti G, Ferreres G. Nonlinear performance of a PI controlled missile: an explanation. International Journal of Robust and Nonlinear Control 1999; 9:485-518.

4. Angeli D. A Lyapunov approach to incremental stability properties. IEEE Transactions on Automatic Control 2002; 47(3):410-421.

5. Zamani Majid, Tabuada P. Backstepping design for incremental stability. IEEE Transactions on Automatic Control 2011; 56(9):2184-2189.

6. Pavlov A, van de Wouw N, Nijmeijer H. Uniform Output Regulation of Nonlinear Systems: A Convergent Dynamics Approach, Systems \& Control: Foundations and Applications. Birkhäuser: Boston, 2006.

7. Wang W, Slotine JJE. On partial contraction analysis for coupled nonlinear oscillators. Biological Cybernetics 2005; 92(1):38-53.

8. Pogromsky AYu. Passivity based design of synchronizing systems. International Journal of Bifurcation and Chaos $1998 ; \mathbf{8}(2): 295-319$.

9. Heemels WPMH, Camlibel MK, Schumacher JM, Brogliato B. Observer-based control of linear complementarity systems. International Journal of Robust and Nonlinear Control 2011; 21(10):1193-1218.

10. Yakubovich VA. Matrix inequalities method in stability theory for nonlinear control systems: I. Absolute stability of forced vibrations. Automation and Remote Control 1964; 7:905-917.

11. Pliss VA. Nonlocal Problems of the Theory of Oscillations. Academic Press: London, 1966.

12. Demidovich BP. Lectures on Stability Theory (In Russian). Nauka: Moscow, 1967.

13. Rüffer BS, van de Wouw N, Mueller M. Convergent systems vs. incremental stability. Systems \& Control Letters 2013; 62(3):277-285.

14. Moreau JJ. Unilateral contact and dry friction in finite freedom dynamics. In Non-smooth Mechanics and Applications, vol. 302, Moreau JJ, Panagiotopoulos PD (eds)., CISM Courses and Lectures. Springer-Verlag: Wien, $1988 ; 1-82$.

15. Moreau JJ. Bounded variation in time. In Topics in Nonsmooth Mechanics, Moreau JJ, Panagiotopoulos PD, Strang G (eds). Birkhäuser: Basel, Boston, Berlin, 1988; 1-74.

16. Leine RI, van de Wouw N. Stability and Convergence of Mechanical Systems with Unilateral Constraints, Lecture Notes in Applied and Computational Mechanics, vol. 36. Springer-Verlag: Berlin, 2008.

17. Brogliato B. Nonsmooth Mechanics. Models, Dynamics and Control (2nd edn), Communications and Control Engineering. Springer-Verlag: London, 1999.

18. Acary V, Brogliato B. Numerical Methods for Nonsmooth Dynamical Systems; Applications in Mechanics and Electronics, Lecture Notes in Applied and Computational Mechanics, vol. 35. Springer-Verlag: Berlin, 2008.

19. Glocker Ch. Set-valued Force Laws, Dynamics of Non-smooth Systems, Lecture Notes in Applied Mechanics, vol. 1. Springer-Verlag: Berlin, 2001.

20. Ambrosio L. Variational problems in SBV and image segmentation. Acta Applicandae Mathematicae: An International Survey Journal on Applying Mathematics and Mathematical Applications 1989; 17(1):1-40.

21. Kalman RE. A new approach to linear filtering and prediction problems. Transactions of the ASME-Journal of Basic Engineering 1960; 82:35-45.

22. Luenberger DG. Observers for multivariable systems. IEEE Transactions on Automatic Control 1966; 11(2): 190-197.

23. Khalil HK, Praly L. High-gain observers in nonlinear feedback control. International Journal of Robust and Nonlinear Control 2014; 24(6):993-1015.

24. Carroll TL, Pecora LM. Synchronizing chaotic circuits. IEEE Transactions on Circuits and Systems 1991; 38(4): 453-456.

25. Josic K. Invariant manifolds and synchronization of coupled dynamical systems. Physical Review Letters 1998; 80(14):3053-3056.

26. Nijmeijer H, Mareels I. An observer looks at synchronisation. IEEE Transactions on Circuits and Systems I 1997; 44(10):882-890.

27. Pavlov A, Pogromsky AY, van de Wouw N, Nijmeijer H. On convergence properties of piecewise affine systems. International Journal of Control 2007; 80(8):1233-1247.

28. Doris A, Juloski AL, Mihajlovic N, Heemels WPMH, van de Wouw N, Nijmeijer H. Observer designs for experimental non-smooth and discontinuous systems. IEEE Transactions on Control Systems Technology 2008; 16(6): 1323-1332.

29. Brogliato B, Heemels WPMH. Observer design for Lur'e systems with multivalued mappings: a passivity approach. IEEE Transactions on Automatic Control 2009; 54(8):1996-2001.

30. Sanfelice RG, Biemond JJB, van de Wouw N, Heemels WPMH. Tracking control for hybrid systems via embedding of known reference trajectories. American Control Conference 2011:869-874.

31. Menini L, Tornambè A. Asymptotic tracking of periodic trajectories for a simple mechanical system subject to nonsmooth impacts. IEEE Transactions on Automatic Control 2001; 46(7):1122-1126. 
32. Biemond JJB, van de Wouw N, Heemels WPMH, Nijmeijer H. Tracking control for hybrid systems with statetriggered jumps. IEEE Transactions on Automatic Control 2013; 58(4):876-890.

33. Menini L, Tornambè A. Velocity observers for non-linear mechanical systems subject to non-smooth impacts. Automatica 2002; 38(12):2169-2175.

34. Martinelli F, Menini L, Tornambè A. Observability, reconstructibility and observer design for linear mechanical systems unobservable in absence of impacts. Journal of Dynamic Systems, Measurement, and Control 2004; 125(4): 549-562.

35. Tanwani A, Brogliato B, Prieur C. Stability and observer design for Lur'e systems with multivalued, nonmonotone, time-varying nonlinearities and state jumps. SIAM Journal on Control and Optimization 2014; 52(6):3639-3672.

36. Camlibel MK, van de Wouw N. On the convergence of linear passive complementarity systems. Proceedings of the 46th IEEE Conference on Decision and Control, New Orleans, USA, 2007; 5886-5891.

37. Pavlov A, Pogromsky A, van de Wouw N, Nijmeijer H. Convergent dynamics, a tribute to Boris Pavlovich Demidovich. Systems and Control Letters 2004; 52(3-4):257-261.

38. Glocker Ch. On frictionless impact models in rigid-body systems. Philosophical Transactions of the Royal Society of London A 2001; 359:2385-2404.

39. Glocker Ch. Energetic consistency conditions for standard impacts. Part I: Newton-type inequality impact laws and Kane's example. Multibody System Dynamics 2013; 29(1):77-117.

40. Glocker Ch. Energetic consistency conditions for standard impacts. Part II: Poisson-type inequality impact laws. Multibody System Dynamics 2013; 32(4):1-65.

41. Leine RI, Baumann M. Variational analysis of inequality impact laws. Proceedings of the 8th EUROMECH Nonlinear Dynamics Conference (ENOC), Vienna, Austria, 2014.

42. Maschek AK. Synchronization of non-smooth mechanical systems. Master's Thesis, ETH Zurich, 2012.

43. Rockafellar RT, Wets RJB. Variational Analysis. Springer-Verlag: Berlin, 1998.

44. Khalil HK. Nonlinear Systems (3rd edn). Prentice Hall: Upper Saddle River, 2002.

45. Paoli L. Continuous dependence on data for vibro-impact problems. Mathematical Models and Methods in Applied Sciences 2005; 15(1):53-93.

46. Horn RA, Johnson CR. Matrix Analysis. Cambridge University Press: Cambridge, 1990

47. Brogliato B. Absolute stability and the Lagrange-Dirichlet theorem with monotone multivalued mappings. Systems \& Control Letters 2004; 51:343-353.

48. Raff T, Allgöwer F. An observer that converges in finite time due to measurement-based state updates. Proceedings of 17th IFAC World Congress, 2008; 2693-2695.

49. Smirnov GV. Introduction to the Theory of Differential Inclusions, Graduate Studies in Mathematics, vol. 41. American Mathematical Society: Providence, 2002. 